\title{
How to Design Robust Algorithms using Noisy Comparison Oracle
}

\author{
Raghavendra Addanki \\ UMass Amherst \\ raddanki@cs.umass.edu
}

\author{
Sainyam Galhotra \\ UMass Amherst \\ sainyam@cs.umass.edu
}

\author{
Barna Saha \\ UC Berkeley \\ barnas@berkeley.edu
}

\begin{abstract}
Metric based comparison operations such as finding maximum, nearest and farthest neighbor are fundamental to studying various clustering techniques such as $k$-center clustering and agglomerative hierarchical clustering. These techniques crucially rely on accurate estimation of pairwise distance between records. However, computing exact features of the records, and their pairwise distances is often challenging, and sometimes not possible. We circumvent this challenge by leveraging weak supervision in the form of a comparison oracle that compares the relative distance between the queried points such as 'Is point $u$ closer to $v$ or $w$ closer to $x$ ?'.

However, it is possible that some queries are easier to answer than others using a comparison oracle. We capture this by introducing two different noise models called adversarial and probabilistic noise. In this paper, we study various problems that include finding maximum, nearest/farthest neighbor search under these noise models. Building upon the techniques we develop for these comparison operations, we give robust algorithms for $k$-center clustering and agglomerative hierarchical clustering. We prove that our algorithms achieve good approximation guarantees with a high probability and analyze their query complexity. We evaluate the effectiveness and efficiency of our techniques empirically on various real-world datasets.
\end{abstract}

\section{PVLDB Reference Format:}

Raghavendra Addanki, Sainyam Galhotra, Barna Saha. How to Design Robust Algorithms using Noisy Comparison Oracle. PVLDB, 14(9): XXX-XXX, 2021. doi:XX.XX/XXX.XX

\section{INTRODUCTION}

Many real world applications such as data summarization, social network analysis, facility location crucially rely on metric based comparative operations such as finding maximum, nearest neighbor search or ranking. As an example, data summarization aims to identify a small representative subset of the data where each representative is a summary of similar records in the dataset. Popular clustering algorithms such as $k$-center clustering and hierarchical clustering are often used for data summarization [25, 39]. In this paper, we study fundamental metric based operations such as finding maximum, nearest neighbor search, and use the developed techniques to study clustering algorithms such as $k$-center clustering and agglomerative hierarchical clustering.

Clustering is often regarded as a challenging task especially due to the absence of domain knowledge, and the final set of clusters

This work is licensed under the Creative Commons BY-NC-ND 4.0 International License. Visit https://creativecommons.org/licenses/by-nc-nd/4.0/ to view a copy of this license. For any use beyond those covered by this license, obtain permission by emailing info@vldb.org. Copyright is held by the owner/author(s). Publication rights licensed to the VLDB Endowment. Proceedings of the VLDB Endowment, Vol. 14, No 9 ISSN 2150-8097. doi:XX.XX/XXX.XX

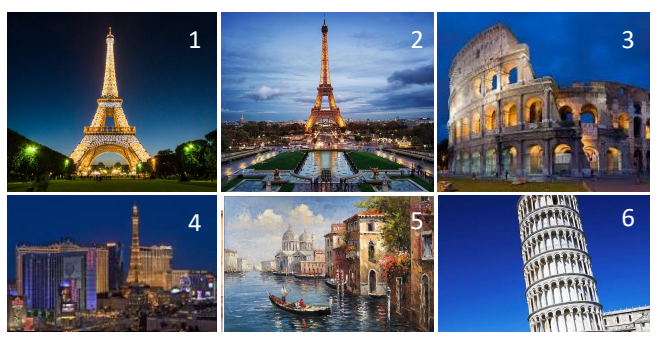

Figure 1: Data summarization example

identified can be highly inaccurate and noisy [7]. It is often hard to compute the exact features of points and thus pairwise distance computation from these feature vectors could be highly noisy. This will render the clusters computed based on objectives such as $k$ center unreliable.

To address these challenges, there has been a recent interest to leverage supervision from crowd workers (abstracted as an oracle) which provides significant improvement in accuracy but at an added cost incurred by human intervention $[20,55,57]$. For clustering, the existing literature on oracle based techniques mostly use optimal cluster queries, that ask questions of the form 'do the points $\mathrm{u}$ and v belong to the same optimal cluster?'[6, 17, 42, 57]. The goal is to minimize the number of queries aka query complexity while ensuring high accuracy of clustering output. This model is relevant for applications where the oracle (human expert or a crowd worker) is aware of the optimal clusters such as in entity resolution [20, 55]. However, in most applications, the clustering output depends highly on the required number of clusters and the presence of other records. Without a holistic view of the entire dataset, answering optimal queries may not be feasible for any realistic oracle. Let us consider an example data summarization task that highlights some of the challenges.

EXAMPLE 1.1. Consider a data summarization task over a collection of images (shown in Figure 1). The goal is to identify $k$ images (say $k=3$ ) that summarize the different locations in the dataset. The images 1,2 refer to the Eiffel tower in Paris, 3 is the Colosseum in Rome, 4 is the replica of Eiffel tower at Las Vegas, USA, 5 is Venice and 6 is the Leaning tower of Pisa. The ground truth output in this case would be $\{\{1,2\},\{3,5,6\},\{4\}\}$. We calculated pairwise similarity between images using the visual features generated from Google Vision API [1]. The pair $(1,4)$ exhibits the highest similarity of 0.87 , while all other pairs have similarity lower than 0.85 . Distance between a pair of images $u$ and $v$, denoted as $d(u, v)$, is defined as (1-similarity between $u$ and $v)$. We ran a user experiment by querying crowd workers to answer simple Yes/No questions to help summarize the data (Please refer to Section 6.2 for more details).

In this example, we make the following observations. 
- Automated clustering techniques generate noisy clusters. Consider the greedy approach for $k$-center clustering [27] which sequentially identifies the farthest record as a new cluster center. In this example, records 1 and 4 are placed in the same cluster by the greedy $k$-center clustering, thereby leading to poor performance. In general, automated techniques are known to generate erroneous similarity values between records due to missing information or even presence of noise [19, 56, 58]. Even Google's landmark detection API [1] did not identify the location of images 4 and 5.

- Answering pairwise optimal cluster query is infeasible. Answering whether 1 and 3 belong to the same optimal cluster when presented in isolation is impossible unless the crowd worker is aware of other records present in the dataset, and the granularity of the optimum clusters. Using the pair-wise Yes/No answers obtained from the crowd workers for the $\left(\begin{array}{c}6 \\ 2\end{array}\right)$ pairs in this example, the identified clusters achieved $0.40 \mathrm{~F}$-score for $k=3$. Please refer to Section 6.2 for additional details.

- Comparing relative distance between the locations is easy. Answering relative distance queries of the form 'Is 1 closer to 3 , or is 5 closer to 6?' does not require any extra knowledge about other records in the dataset. For the 6 images in the example, we queried relative distance queries and the final clusters constructed for $k=3$ achieved an F-score of 1 .

In summary, we observe that humans have an innate understanding of the domain knowledge and can answer relative distance queries between records easily. Motivated by the aforementioned observations, we consider a quadruplet comparison oracle that compares the relative distance between two pairs of points $\left(u_{1}, u_{2}\right)$ and $\left(v_{1}, v_{2}\right)$ and outputs the pair with smaller distance between them breaking ties arbitrarily. Such oracle models have been studied extensively in the literature [11, 17, 24, 32, 34, 48, 49]. Even though quadruplet queries are easier than binary optimal queries, some oracle queries maybe harder than the rest. In a comparison query, if there is a significant gap between the two distances being compared, then such queries are easier to answer $[9,15]$. However, when the two distances are close, the chances of an error could increase. For example, 'Is location in image 1 closer to 3 , or 2 is closer to 6?' maybe difficult to answer.

To capture noise in quadruplet comparison oracle answers, we consider two noise models. In the first noise model, when the pairwise distances are comparable, the oracle can return the pair of points that are farther instead of closer. Moreover, we assume that the oracle has access to all previous queries and can answer queries by acting adversarially. More formally, there is a parameter $\mu>0$ such that if $\frac{\max d\left(u_{1}, u_{2}\right), d\left(v_{1}, v_{2}\right)}{\min d\left(u_{1}, u_{2}\right), d\left(v_{1}, v_{2}\right)} \leq(1+\mu)$, then adversarial error may occur, otherwise the answers are correct. We call this the "Adversarial Noise Model". In the second noise model called "Probabilistic Noise Model", given a pair of distances, we assume that the oracle answers correctly with a probability of $1-p$ for some fixed constant $p<\frac{1}{2}$. We consider a persistent probabilistic noise model, where our oracle answers are persistent, i.e., query responses remain unchanged even upon repeating the same query multiple times. Such noise models have been studied extensively [9, 10, 20, 24, 42, 46] since the error due to oracles often does not change with repetition, and in some cases increases upon repeated querying [20, 42, 46].
This is in contrast to the noise models studied in [17] where response to every query is independently noisy. Persistent query models are more difficult to handle than independent query models where repeating each query is sufficient to generate the correct answer by majority voting.

\subsection{Our Contributions}

We present algorithms for finding the maximum, nearest and farthest neighbors, $k$-center clustering and hierarchical clustering objectives under the adversarial and probabilistic noise model using comparison oracle. We show that our techniques have provable approximation guarantees for both the noise models, are efficient and obtain good query complexity. We empirically evaluate the robustness and efficiency of our techniques on real world datasets. (i) Maximum, Farthest and Nearest Neighbor: Finding maximum has received significant attention under both adversarial and probabilistic models $[4,9,15,18,21-23,38]$. In this paper, we provide the following results.

- Maximum under adversarial model. We present an algorithm that returns a value within $(1+\mu)^{3}$ of the maximum among a set of $n$ values $V$ with probability $1-\delta^{1}$ using $O\left(n \log ^{2}(1 / \delta)\right)$ oracle queries and running time (Theorem 3.6).

- Maximum under probabilistic model. We present an algorithm that requires $O\left(n \log ^{2}(n / \delta)\right)$ queries to identify $O\left(\log ^{2}(n / \delta)\right)$ th rank value with probability $1-\delta$ (Theorem 3.7$)$. In other words, in $O\left(n \log ^{2}(n)\right)$ time we can identify $O\left(\log ^{2}(n)\right)$ th value in the sorted order with probability $1-\frac{1}{n^{c}}$ for any constant $c$. To contrast our results with the state of the art, Ajtai et al. [4] study a slightly different additive adversarial error model where the answer of a maximum query is correct if the compared values differ by $\theta$ (for some $\theta>0$ ) and otherwise the oracle answers adversarially. Under this setting, they give an additive $3 \theta$-approximation with $O(n)$ queries. Although, our model cannot be directly compared with theirs, we note that our model is scale invariant, and thus, provides a much stronger bound when distances are small. As a consequence, our algorithm can be used under additive adversarial model as well, and obtaining the same approximation guarantees (Theorem 3.10).

For the probabilistic model, after a long series of works [9, 21, 23, 38 ], only recently an algorithm is proposed with query complexity $O(n \log n)$ that returns an $O(\log n)$ th rank value with probability $1-\frac{1}{n}$ [22]. Previously, the best query complexity was $O\left(n^{3 / 2}\right)$ [23]. While our bounds are slightly worse than [22], our algorithm is significantly simpler.

Rest of the work in finding maximum allow repetition of queries and assume the answers are independent $[15,18]$. As discussed earlier, persistent errors are much more difficult to handle than independent errors. In [18], the authors present an algorithm that finds maximum using $O(n \log 1 / \delta)$ queries and succeeds with probability $1-\delta$. Therefore, even under persistent errors, we obtain guarantees close to the existing ones which assume independent error. The algorithms of $[15,18]$ do not extend to our model.

- Nearest Neighbor. Nearest neighbor queries can be cast as "finding minimum" among a set of distances. We can obtain bounds

${ }^{1} \delta$ is the confidence parameter and is standard in the literature of randomized algorithms. 
similar to finding maximum for the nearest neighbor queries. In the adversarial model, we obtain an $(1+\mu)^{3}$-approximation, and in the probabilistic model, we are guaranteed to return an element with rank $O\left(\log ^{2}(n / \delta)\right)$ with probability $1-\delta$ using $O\left(n \log ^{2}(1 / \delta)\right)$ and $O\left(n \log ^{2}(n / \delta)\right)$ oracle queries respectively.

Prior techniques have studied nearest neighbor search under noisy distance queries [41], where the oracle returns a noisy estimate of a distance between queried points, and repetitions are allowed. Neither the algorithm of [41], nor other techniques developed for maximum $[4,18]$ and top- $k$ [15] extend for nearest neighbor under our noise models.

- Farthest Neighbor. Similarly, the farthest neighbor query can be cast as finding maximum among a set of distances, and the results for computing maximum extends to this setting. However, computing the farthest neighbor is one of the basic primitives for more complex tasks like $k$-center clustering, and for that, the existing bounds under the probabilistic model that return an $O(\log n)$ th rank element is insufficient. Since distances on a metric space satisfies triangle inequality, we exploit it to get a constant approximation to the farthest query under the probabilistic model and a mild distribution assumption (Theorem 3.10).

(ii) $k$-center Clustering: $k$-center clustering is one of the fundamental models of clustering and is very well-studied [52,59].

- $k$-center under adversarial model We design algorithm that returns a clustering that is a $2+\mu$ approximation for small values of $\mu$ with probability $1-\delta$ using $O\left(n k^{2}+n k \log ^{2}(k / \delta)\right)$ queries (Theorem 4.2). In contrast, even when exact distances are known, $k$ center cannot be approximated better than a 2-factor unless $P=N P$ [52]. Therefore, we achieve near-optimal results.

- $k$-center under probabilistic noise model. For probabilistic noise, when optimal $k$-center clusters are of size at least $\Omega(\sqrt{n})$, our algorithm returns a clustering that achieves constant approximation with probability $1-\delta$ using $O\left(n k \log ^{2}(n / \delta)\right)$ queries (Theorem 4.4)

To the best of our knowledge, even though $k$-center clustering is an extremely popular and basic clustering paradigm, it hasn't been studied under the comparison oracle model, and we provide the first results in this domain.

(iii) Single Linkage and Complete Linkage- Agglomerative Hierarchical Clustering : Under adversarial noise, we show a clustering technique that loses only a multiplicative factor of $(1+\mu)^{3}$ in each merge operation and has an overall query complexity of $O\left(n^{2}\right)$. Prior work [24] considers comparison oracle queries to perform average linkage in which the unobserved pairwise similarities are generated according to a normal distribution. These techniques do not extend to our noise models.

\subsection{Other Related Work}

For finding the maximum among a given set of values, it is known that techniques based on tournament obtain optimal guarantees and are widely used [15]. For the problem of finding nearest neighbor, techniques based on locality sensitive hashing generally work well in practice [5]. Clustering points using $k$-center objective is NPhard and there are many well known heuristics and approximation algorithms [59] with the classic greedy algorithm achieving an approximation ratio of 2 . All these techniques are not applicable when pairwise distances are unknown. As distances between points cannot always be accurately estimated, many recent techniques leverage supervision in the form of an oracle. Most oracle based clustering frameworks consider 'optimal cluster' queries [13, 28, $33,42,43]$ to identify ground truth clusters. Recent techniques for distance based clustering objectives, such as $k$-means $[6,12,36,37]$ and $k$-median [3] use optimal cluster queries in addition to distance information for obtaining better approximation guarantees. As 'optimal cluster' queries can be costly or sometimes infeasible, there has been recent interest in leveraging distance based comparison oracles for other problems similar to our quadruplet oracles [17, 24].

Distance based comparison oracles have been used to study a wide range of problems and we list a few of them - learning fairness metrics [34], top-down hierarchical clustering with a different objective [11, 17, 24], correlation clustering [49] and classification $[32,48]$, identify maximum $[30,53]$, top- $k$ elements $[14-$ 16, 38, 40, 45], information retrieval [35], skyline computation [54]. To the best of our knowledge, there is no work that considers quadruplet comparison oracle queries to perform $k$-center clustering and single/complete linkage based hierarchical clustering.

Closely related to finding maximum, sorting has also been well studied under various comparison oracle based noise models [8, 9]. The work of [15] considers a different probabilistic noise model with error varying as a function of difference in the values but they assume that each query is independent and therefore repetition can help boost the probability of success. Using a quadruplet oracle, [24] studies the problem of recovering a hierarchical clustering under a planted noise model and is not applicable for single linkage.

\section{PRELIMINARIES}

Let $V=\left\{v_{1}, v_{2}, \ldots, v_{n}\right\}$ be a collection of $n$ records such that each record maybe associated with a value $\operatorname{val}\left(v_{i}\right), \forall i \in[1, n]$. We assume that there exists a total ordering over the values of elements in $V$. For simplicity we denote the value of record $v_{i}$ as $v_{i}$ instead of $\operatorname{val}\left(v_{i}\right)$ whenever it is clear from the context.

Given this setting, we consider a comparison oracle that compares the values of any pair of records $\left(v_{i}, v_{j}\right)$ and outputs Yes if $v_{i} \leq v_{j}$ and No otherwise.

DeFinition 2.1 (COMPARISON ORACle). An oracle is a function $O: V \times V \rightarrow\{$ Yes, No\}. Each oracle query considers two values as input and outputs $O\left(v_{1}, v_{2}\right)=$ Yes if $v_{1} \leq v_{2}$ and No otherwise.

Note that a comparison oracle is defined for any pair of values. Given this oracle setting, we define the problem of identifying the maximum over the records $V$.

Problem 2.2 (MAхімum). Given a collection of $n$ records $V=$ $\left\{v_{1}, \ldots, v_{n}\right\}$ and access to a comparison oracle $O$, identify the $\arg \max _{v_{i} \in V} v_{i}$ with minimum number of queries to the oracle.

As a natural extension, we can also study the problem of identifying the record corresponding to the smallest value in $V$.

\subsection{Quadruplet Oracle Comparison Query}

In applications that consider distance based comparison of records like nearest neighbor identification, the records $V=\left\{v_{1}, \ldots, v_{n}\right\}$ are generally considered to be present in a high-dimensional metric space along with a distance $d: V \times V \rightarrow \mathbb{R}^{+}$defined over pairs of 
records. We assume that the embedding of records in latent space is not known, but there exists an underlying ground truth [5]. Prior techniques mostly assume complete knowledge of accurate distance metric and are not applicable in our setting. In order to capture the setting where we can compare distances between pair of records, we define quadruplet oracle below.

Definition 2.3 ( Quadruplet Oracle). An oracle is a function $O: V \times V \times V \times V \rightarrow\{$ Yes, No\}. Each oracle query considers two pairs of records as input and outputs $O\left(v_{1}, v_{2}, v_{3}, v_{4}\right)=$ Yes if $d\left(v_{1}, v_{2}\right) \leq$ $d\left(v_{3}, v_{4}\right)$ and No otherwise.

The quadruplet oracle is similar to the comparison oracle discussed before with a difference that the two values being compared are associated with pair of records as opposed to individual records. Given this oracle setting, we define the problem of identifying the farthest record over $V$ with respect to a query point $q$ as follows.

Problem 2.4 (FARthest Point). Given a collection of $n$ records $V=\left\{v_{1}, \ldots, v_{n}\right\}$, a query record $q$ and access to a quadruplet oracle $O$, identify $\arg \max _{v_{i} \in V \backslash\{q\}} d\left(q, v_{i}\right)$.

Similarly, the nearest neighbor query returns a point that satisfies $\arg \min _{u_{i} \in V \backslash\{q\}} d\left(q, u_{i}\right)$. Now, we formally define the k-center clustering problem.

Problem 2.5 (K-Center clustering). Given a collection of $n$ records $V=\left\{v_{1}, \ldots, v_{n}\right\}$ and access to a comparison oracle $O$, identify $k$ centers (say $S \subseteq V$ ) and a mapping of records to corresponding centers, $\pi: V \rightarrow S$, such that the maximum distance of any record from its center, i.e., $\max _{v_{i} \in V} d\left(v_{i}, \pi\left(v_{i}\right)\right)$ is minimized.

We assume that the points $v_{i} \in V$ exist in a metric space and the distance between any pair of points is not known. We denote the unknown distance between any pair of points $\left(v_{i}, v_{j}\right)$ where $v_{i}, v_{j} \in V$ as $d\left(v_{i}, v_{j}\right)$ and use $k$ to denote the number of clusters. Optimal clusters are denoted as $C^{*}$ with $C^{*}\left(v_{i}\right) \subseteq V$ denoting the set of points belonging to the optimal cluster containing $v_{i}$. Similarly, $C\left(v_{i}\right) \subseteq V$ refers to the nodes belonging to the cluster containing $v_{i}$ for any clustering given by $C(\cdot)$.

In addition to the k-center clustering, we study single linkage and complete linkage-agglomerative clustering techniques where the distance metric over the records is not known apriori. These techniques initialize each record $v_{i}$ in a separate singleton cluster and sequentially merge the pair of clusters having the least distance between them. In case of single linkage, the distance between two clusters $C_{1}$ and $C_{2}$ is characterized by the closest pair of records defined as:

$$
d_{S L}\left(C_{1}, C_{2}\right)=\min _{v_{i} \in C_{1}, v_{j} \in C_{2}} d\left(v_{i}, v_{j}\right)
$$

In complete linkage, the distance between a pair of clusters $C_{1}$ and $C_{2}$ is calculated by identifying the farthest pair of records, $d_{C L}\left(C_{1}, C_{2}\right)=\max _{v_{i} \in C_{1}, v_{j} \in C_{2}} d\left(v_{i}, v_{j}\right)$.

\subsection{Noise Models}

The oracle models discussed in Problem 2.2, 2.4 and 2.5 assume that the oracle answers every comparison query correctly. In real world applications, however, the answers can be wrong which can lead to noisy results. To formalize the notion of noise, we consider two different models. First, adversarial noise model considers a setting where a comparison query can be adversarially wrong if the two values being compared are within a multiplicative factor of $(1+\mu)$ for some constant $\mu>0$.

$$
O\left(v_{1}, v_{2}\right)=\left\{\begin{array}{l}
\text { Yes, if } v_{1}<\frac{1}{(1+\mu)} v_{2} \\
\text { No, if } v_{1}>(1+\mu) v_{2} \\
\text { adversarially incorrect if } \frac{1}{(1+\mu)} \leq \frac{v_{1}}{v_{2}} \leq(1+\mu)
\end{array}\right.
$$

The parameter $\mu$ corresponds to the degree of error. For example, $\mu=0$ implies a perfect oracle. The model extends to quadruplet oracle as follows.

$O\left(v_{1}, v_{2}, v_{3}, v_{4}\right)=\left\{\begin{array}{l}\text { Yes, if } d\left(v_{1}, v_{2}\right)<\frac{1}{(1+\mu)} d\left(v_{3}, v_{4}\right) \\ \text { No, if } d\left(v_{1}, v_{2}\right)>(1+\mu) d\left(v_{3}, v_{4}\right) \\ \text { adversarially incorrect if } \frac{1}{(1+\mu)} \leq \frac{d\left(v_{1}, v_{2}\right)}{d\left(v_{3}, v_{4}\right)} \leq(1+\mu)\end{array}\right.$

The second model considers a probabilistic noise model where each comparison query is incorrect independently with a probability $p<\frac{1}{2}$ and asking the same query multiple times yields the same response. We discuss ways to estimate $\mu$ and $p$ from real data in Section 6.

\section{FINDING MAXIMUM}

In this section, we present robust algorithms to identify the record corresponding to the maximum value in $V$ under the adversarial noise model and the probabilistic noise model. Later we extend the algorithms to find the farthest and the nearest neighbor. We note that our algorithms for the adversarial model are parameter free (do not depend on $\mu$ ) and the algorithms for the probabilistic model can use $p=0.5$ as a worst case estimate of the noise.

\subsection{Adversarial Noise}

Consider a trivial approach that maintains a running maximum value while sequentially processing the records, i.e., if a larger value is encountered, the current maximum value is updated to the larger value. This approach requires $n-1$ comparisons. However, in the presence of adversarial noise, our output can have a significantly lower value compared to the correct maximum. In general, if $v_{\max }$ is the true maximum of $V$, then the above approach can return an approximate maximum whose value could be as low as $v_{\max } /(1+\mu)^{n-1}$. To see this, assume $v_{1}=1$, and $v_{i}=(1+\mu-\epsilon)^{i}$ where $\epsilon>0$ is very close to 0 . It is possible that while comparing $v_{i}$ and $v_{i+1}$, the oracle returns $v_{i}$ as the larger element. If this mistake is repeated for every $i$, then, $v_{1}$ will be declared as the maximum element whereas the correct answer is $v_{n} \approx v_{1}(1+\mu)^{n-1}$.

To improve upon this naive strategy, we introduce a natural keeping score based idea where given a set $S \subseteq V$ of records, we maintain Count $(v, S)$ that is equal to the number of values smaller than $v$ in $S$.

$$
\operatorname{Count}(v, S)=\sum_{x \in S \backslash\{v\}} 1\{O(v, x)==\mathrm{No}\}
$$

It is easy to observe that when the oracle makes no mistakes, Count $\left(s_{\max }, S\right)=|S|-1$ and obtains the highest score, where $s_{\max }$ is the maximum value in $S$. Using this observation, in Algorithm 1, we output the value with the highest Count score. 
Given a set of records $V$, we show in Lemma 3.1 that Count-Max $(V)$ obtained using Algorithm 1 always returns a good approximation of the maximum value in $V$.

Lemma 3.1. Given a set of values $V$ with maximum value $v_{\max }$, $\operatorname{CoUNT-MAX}(V)$ returns a value $u_{\max }$ where $u_{\max } \geq v_{\max } /(1+\mu)^{2}$ using $O\left(|V|^{2}\right)$ oracle queries.

Using Example 3.2, when $\mu=1$, we demonstrate that $(1+\mu)^{2}=4$ approximation ratio is achieved by Algorithm 1.

EXAMPLE 3.2. Let $S$ denote a set of four records $u, v, w$ and $t$ with ground truth values 51,101, 102 and 202, respectively. While identifying the maximum value under adversarial noise with $\mu=1$, the oracle must return a correct answer to $O(u, t)$ and all other oracle query answers can be incorrect adversarially. If the oracle answers all other queries incorrectly, we have, Count values of $t, w, u, v$ are $1,1,2$, and 2 respectively. Therefore, $u$ and $v$ are equally likely, and when Algorithm 1 returns $u$, we have a $202 / 51 \approx 3.96$ approximation.

From Lemma 3.1, we have that $O\left(n^{2}\right)$ oracle queries where $|V|=n$, are required to get $(1+\mu)^{2}$ approximation. In order to improve the query complexity, we use a tournament to obtain the maximum value. The idea of using a tournament for finding maximum has been studied in the past $[15,18]$.

Algorithm 2 presents pseudo code of the approach that takes values $V$ as input and outputs an approximate maximum value. It constructs a balanced $\lambda$-ary tree $\mathcal{T}$ containing $n$ leaf nodes such that a random permutation of the values $V$ is assigned to the leaves of $\mathcal{T}$. In a tournament, the internal nodes of $\mathcal{T}$ are processed bottomup such that at every internal node $w$, we assign the value that is largest among the children of $w$. To identify the largest value, we calculate $\arg \max _{v \in \operatorname{children}(w)} \operatorname{Count}(v$, children $(w))$ at the internal node $w$, where Count $(v, X)$ refers to the number of elements in $X$ that are considered smaller than $v$. Finally, we return the value at the root of $\mathcal{T}$ as our output. In Lemma 3.3, we show that Algorithm 2 returns a value that is a $(1+\mu)^{2 \log _{\lambda} n}$ multiplicative approximation of the maximum value.

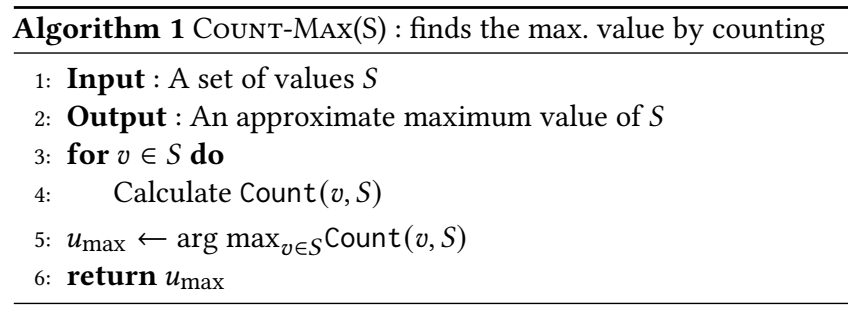

Lemma 3.3. Suppose $v_{\max }$ is the maximum value among the set of records $V$. Algorithm 2 outputs a value $u_{\max }$ such that $u_{\max } \geq$ $\frac{v_{\max }}{(1+\mu)^{\log _{\lambda} n}}$ using $O(n \lambda)$ oracle queries.

According to Lemma 3.3, Algorithm 2 identifies a constant approximation when $\lambda=\Theta(n), \mu$ is a fixed constant and has a query complexity of $\Theta\left(n^{2}\right)$. By reducing the degree of the tournament tree from $\lambda$ to 2 , we can achieve $\Theta(n)$ query complexity, but with a worse approximation ratio of $(1+\mu)^{\log n}$.

Now, we describe our main algorithm (Algorithm 4) that uses the the following observation to improve the overall query complexity.
ObSERvation 3.4. At an internal node $w \in \mathcal{T}$, the identified maximum is incorrect only if there exists $x \in$ children ( $w)$ that is very close to the true maximum (say $w_{\max }$ ), i.e. $\frac{w_{\max }}{(1+\mu)} \leq x \leq(1+\mu) w_{\max }$.

Based on the above observation, our Algorithm MAX-ADv uses two steps to identify a good approximation of $v_{\max }$. Consider the case when there are a lot of values that are close to $v_{\max }$. In Algorithm MAX-ADV, we use a subset $\widetilde{V} \subseteq V$ of size $\sqrt{n} t$ (for a suitable choice of parameter $t$ ) obtained using uniform sampling with replacement. We show that using a sufficiently large subset $\widetilde{V}$, obtained by sampling, we ensure that at least one value that is closer to $v_{\max }$ is in $\widetilde{V}$, thereby giving a good approximation of $v_{\max }$.

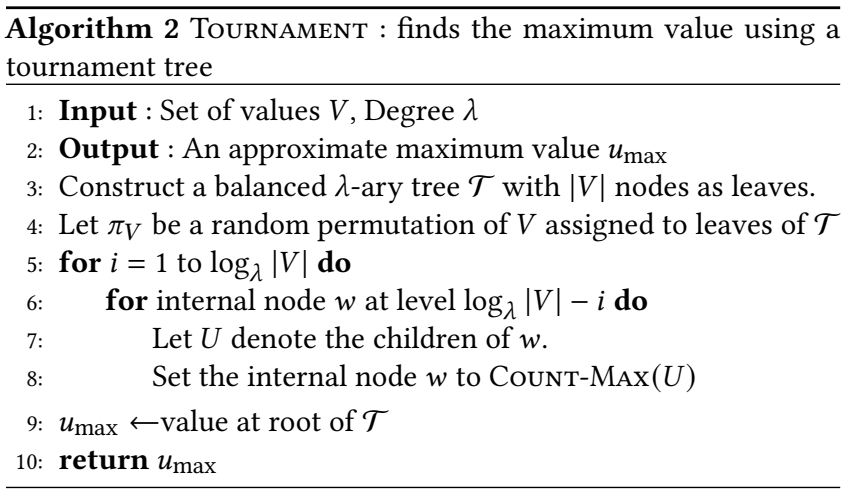

In order to handle the case when there are only a few values closer to $v_{\max }$, we divide the entire data set into $l$ disjoint parts (for a suitable choice of $l$ ) and run the TournAmEnT algorithm with degree $\lambda=2$ on each of these parts separately (Algorithm 3). As there are very few points close to $v_{\max }$, the probability of comparing any such value with $v_{\max }$ is small, and this ensures that in the partition containing $v_{\max }$, TouRnAMENT returns $v_{\text {max }}$. We collect the maximum values returned by Algorithm 2 from all the partitions and include these values in $T$ in Algorithm MAx-ADv. We repeat this procedure $t$ times and set $l=\sqrt{n}, t=2 \log (2 / \delta)$ to achieve the desired success probability $1-\delta$. We combine the outputs of both the steps, i.e., $\widetilde{V}$ and $T$ and output the maximum among them using Count-Max. This ensures that we get a good approximation as we use the best of both the approaches.

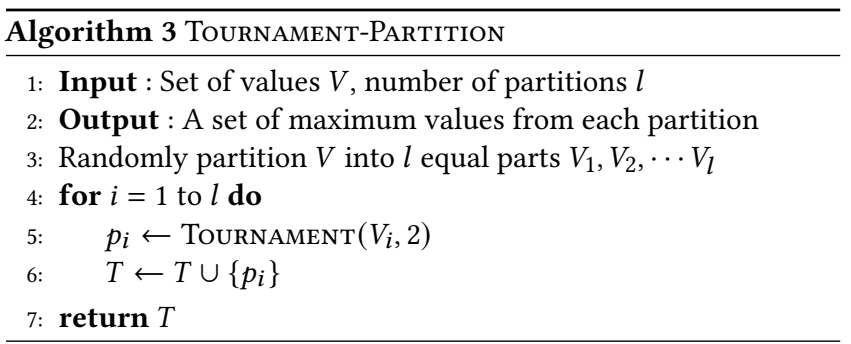

Theoretical Guarantees. In order to prove approximation guarantee of Algorithm 4, we first argue that the sample $\widetilde{V}$ contains a good approximation of the maximum value $v_{\max }$ with a high probability. Let $C$ denote the set of values that are very close to $v_{\max }$. Suppose $C=\left\{u: v_{\max } /(1+\mu) \leq u \leq v_{\max }\right\}$. In Lemma 3.5, we first 


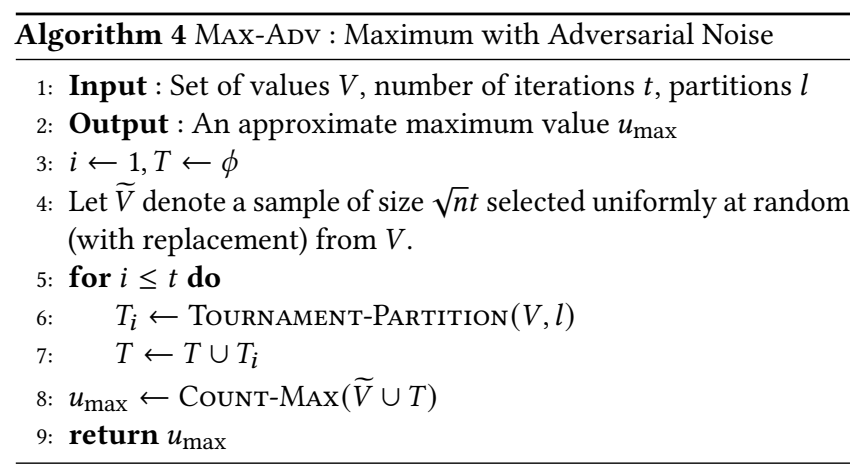

show that $\widetilde{V}$ contains a value $v_{j} \in \widetilde{V}$ such that $v_{j} \geq v_{\max } /(1+\mu)$, whenever the size of $C$ is large, i.e., $|C|>\sqrt{n} / 2$. Otherwise, we show that we can recover $v_{\max }$ correctly with probability $1-\delta / 2$ whenever $|C| \leq \sqrt{n} / 2$.

Lemma 3.5. (1) If $|C|>\sqrt{n} / 2$, then there exists a value $v_{j} \in \widetilde{V}$ satisfying $v_{j} \geq v_{\max } /(1+\mu)$ with probability of $1-\delta / 2$.

(2) Suppose $|C| \leq \sqrt{n} / 2$. Then, $T$ contains $v_{\max }$ with probability at least $1-\delta / 2$.

Now, we briefly provide a sketch of the proof of Lemma 3.5 . Consider the first step, where we use a uniformly random sample $\widetilde{V}$ of $\sqrt{n} t$ points from $V$ (obtained with replacement). When $|C| \geq$ $\sqrt{n} / 2$, probability that $\widetilde{V}$ contains a value from $C$ is given by $1-$ $(1-|C| / n)^{|\widetilde{V}|}=1-\left(1-\frac{1}{2 \sqrt{n}}\right)^{2 \sqrt{n} \log (2 / \delta)} \approx 1-\delta / 2$.

In the second step, Algorithm 4 uses a modified tournament tree that partitions the set $V$ into $l=\sqrt{n}$ parts of size $n / l=\sqrt{n}$ each and identifies a maximum $p_{i}$ from each partition $V_{i}$ using Algorithm 2. We have that the expected number of elements from $C$ in a partition $V_{i}$ containing $v_{\max }$ is $|C| / l=\sqrt{n} /(2 \sqrt{n})=1 / 2$. Thus by the Markov's inequality, the probability that $V_{i}$ contains a value from $C$ is $\leq 1 / 2$. With $1 / 2$ probability, $v_{\max }$ will never be compared with any point from $C$ in the partition $V_{i}$. To increase the success probability, we run this procedure $t$ times and obtain all the outputs. Among the $t$ runs of Algorithm 2, we argue that $v_{\max }$ is never compared with any value of $C$ in at least one of the iterations with a probability at least $1-(1-1 / 2)^{2 \log (2 / \delta)} \geq 1-\delta / 2$.

In Lemma 3.1, we show that using CounT-Max we get a $(1+\mu)^{2}$ multiplicative approximation. Combining it with Lemma 3.5, we have that $u_{\max }$ returned by Algorithm 4 satisfies $u_{\max } \geq \frac{v_{\max }}{(1+\mu)^{3}}$ with probability $1-\delta$. For query complexity, Algorithm 3 identifies $\sqrt{n} t$ samples denoted by $\widetilde{V}$. These identified values, along with $T$ are then processed by CounT-MAX to identify the maximum $u_{\text {max }}$. This step requires $O\left(|\widetilde{V} \cup T|^{2}\right)=O\left(n \log ^{2}(1 / \delta)\right)$ oracle queries.

Theorem 3.6. Given a set of values $V$, Algorithm 4 returns a $(1+\mu)^{3}$ approximation of maximum value with probability $1-\delta$ using $O\left(n \log ^{2}(1 / \delta)\right)$ oracle queries.

\subsection{Probabilistic Noise}

We cannot directly extend the algorithms for the adversarial noise model to probabilistic noise. Specifically, the theoretical guarantees of Lemma 3.3 do not apply when the noise is probabilistic. In this section, we develop several new ideas to handle probabilistic noise.

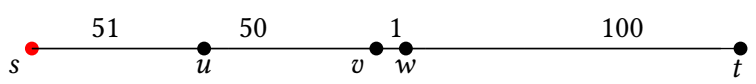

Figure 2: Example for Lemma 3.1 with $\mu=1$.

Let $\operatorname{rank}(u, V)$ denote the index of $u$ in the non-increasing sorted order of values in $V$. So, $v_{\max }$ will have rank 1 and so on. Our main idea is to use an early stopping approach that uses a sample $S \subseteq V$ of $O(\log (n / \delta))$ values selected randomly and for every value $u$ that is not in $S$, we calculate Count $(u, S)$ and discard $u$ using a chosen threshold for the Count scores. We argue that by doing so, it helps us eliminate the values that are far away from the maximum in the sorted ranking. This process is continued $\Theta(\log n)$ times to identify the maximum value. We present the pseudo code in the Appendix and prove the following approximation guarantee.

THEOREM 3.7. There is an algorithm that returns $u_{\max } \in V$ such that $\operatorname{rank}\left(u_{\max }, V\right)=O\left(\log ^{2}(n / \delta)\right)$ with probability $1-\delta$ and requires $O\left(n \log ^{2}(n / \delta)\right)$ oracle queries.

The algorithm to identify the minimum value is same as that of maximum with a modification where Count scores consider the case of Yes (instead of No): Count $(v, S)=\sum_{x \in S \backslash\{v\}} 1\{O(v, x)==$ Yes $\}$

\subsection{Extension to Farthest and Nearest Neighbor}

Given a set of records $V$, the farthest record from a query $u$ corresponds to the record $u^{\prime} \in V$ such that $d\left(u, u^{\prime}\right)$ is maximum. This query is equivalent to finding maximum in the set of distance values given by $D(u)=\left\{d\left(u, u^{\prime}\right) \mid \forall u^{\prime} \in V\right\}$ containing $n$ values for which we already developed algorithms in Section 3. Since the ground truth distance between any pair of records is not known, we require quadruplet oracle (instead of comparison oracle) to identify the maximum element in $D(u)$. Similarly, the nearest neighbor of query record $u$ corresponds to finding the record with minimum distance value in $D(u)$. Algorithms for finding maximum from previous sections, extend for these settings with similar guarantees.

EXAMPLE 3.8. Figure 2 shows a worst-case example for the approximation guarantee to identify the farthest point from $s$ (with $\mu=1$ ). Similar to Example 3.2, we have, Count values of $t, w, u, v$ are $1,1,2,2$ respectively. Therefore, $u$ and $v$ are equally likely, and when Algorithm 1 outputs $u$, we have $a \approx 3.96$ approximation.

For probabilistic noise, the farthest identified in Section 3.2 is guaranteed to rank within the top- $O\left(\log ^{2} n\right)$ values of set $V$ (Theorem 3.7). In this section, we show that it is possible to compute the farthest point within a small additive error under the probabilistic model, if the data set satisfies an additional property discussed below. For the simplicity of exposition, we assume $p \leq 0.40$, though our algorithms work for any value of $p<0.5$ (with different constants).

One of the challenges in developing robust algorithms for farthest identification is that every relative distance comparison of records from $u\left(O\left(u, v_{i}, u, v_{j}\right)\right.$ for some $\left.v_{i}, v_{j} \in V\right)$ may be answered incorrectly with constant error probability $p$ and the success probability cannot be boosted by repetition. We overcome this challenge by performing pairwise comparisons in a robust manner. Suppose the desired failure probability is $\delta$, we observe that if $\Theta(\log (1 / \delta))$ records closest to the query $u$ are known (say 


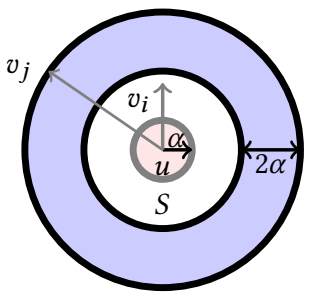

In this example, $O\left(u, v_{i}, u, v_{j}\right)$ is answered correctly with a probability $1-p$. To boost the correctness probability, FCount uses the queries $O\left(x, v_{i}, x, v_{j}\right), \forall x$ in the red region around $u$, denoted by $S$.

Figure 3: Algorithm 5 returns 'Yes' as $d\left(u, v_{i}\right)<d\left(u, v_{j}\right)-2 \alpha$.

$S)$ and $\max _{x \in S}\{d(u, x)\} \leq \alpha$ for some $\alpha>0$, then each pairwise comparison of the form $O\left(u, v_{i}, u, v_{j}\right)$ can be replaced by Algorithm PAIRWISECOMP and use it to execute Algorithm 4. Algorithm 5 takes the two records $v_{i}$ and $v_{j}$ as input along with $S$ and outputs Yes or No where Yes denotes that $v_{i}$ is closer to $u$. We calculate $\operatorname{FCount}\left(v_{i}, v_{j}\right)=\sum_{x \in S} \mathbf{1}\left\{O\left(v_{i}, x, v_{j}, x\right)==\right.$ Yes $\}$ as a robust estimate where the oracle considers $v_{i}$ to be closer to $x$ than $v_{j}$. If FCount $\left(v_{i}, v_{j}\right)$ is smaller than $0.3|S| \leq(1-p)|S| / 2$ then we output No and Yes otherwise. Therefore, every pairwise comparison query is replaced with $\Theta(\log (1 / \delta))$ quadruplet queries using Algorithm 5 .

We argue that Algorithm 5 will output the correct answer with a high probability if $\left|d\left(u, v_{j}\right)-d\left(u, v_{i}\right)\right| \geq 2 \alpha$ (See Fig 3). In Lemma 3.9, we show that, if $d\left(u, v_{j}\right)>d\left(u, v_{i}\right)+2 \alpha$, then, FCount $\left(v_{i}, v_{j}\right) \geq$ $0.3|S|$ with probability $1-\delta$.

Lemma 3.9. Suppose $\max _{v_{i} \in S} d\left(u, v_{i}\right) \leq \alpha$ and $|S| \geq 6 \log (1 / \delta)$. Consider two records $v_{i}$ and $v_{j}$ such that $d\left(u, v_{i}\right)<d\left(u, v_{j}\right)-2 \alpha$ then FCount $\left(v_{i}, v_{j}\right) \geq 0.3|S|$ with a probability of $1-\delta$.

With the help of Algorithm 5, relative distance query of any pair of records $v_{i}, v_{j}$ from $u$ can be answered correctly with a high probability provided $\left|d\left(u, v_{i}\right)-d\left(u, v_{j}\right)\right| \geq 2 \alpha$. Therefore, the output of Algorithm 5 is equivalent to an additive adversarial error model where any quadruplet query can be adversarially incorrect if the distance $\left|d\left(u, v_{i}\right)-d\left(u, v_{j}\right)\right|<2 \alpha$ and correct otherwise. In the Appendix, we show that Algorithm 4 can be extended to the additive adversarial error model, such that each comparison $\left(u, v_{i}, u, v_{j}\right)$ is replaced by PAIRWISECOMP (Algorithm 5). We give an approximation guarantee, that loses an additive $6 \alpha$ following a similar analysis of Theorem 3.6.

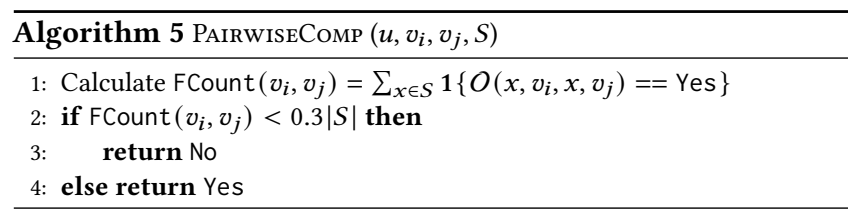

Theorem 3.10. Given a query vertex $u$ and $a$ set $S$ with $|S|=$ $\Omega(\log (n / \delta))$ such that $\max _{v \in S} d(u, v) \leq \alpha$ then the farthest identified using Algorithm 4 (with PAIRWISECOMP), denoted by $u_{\text {max }}$ is within $6 \alpha$ distance from the optimal farthest point, i.e., $d\left(u, u_{\max }\right) \geq$ $\max _{v \in V} d(u, v)-6 \alpha$ with a probability of $1-\delta$. Further the query complexity is $O\left(n \log ^{3}(n / \delta)\right)$.

\section{$4 \quad k$-CENTER CLUSTERING}

In this section, we present algorithms for $k$-center clustering and prove constant approximation guarantees of our algorithm. Our algorithm is an adaptation of the classical greedy algorithm for $k$-center [27]. The greedy algorithm [27] is initialized with an arbitrary point as the first cluster center and then iteratively identifies the next centers. In each iteration, it assigns all the points to the current set of clusters, by identifying the closest center for each point. Then, it finds the farthest point among the clusters and uses it as the new center. This technique requires $O(n k)$ distance comparisons in the absence of noise and guarantees 2-approximation of the optimal clustering objective. We provide the pseudocode for this approach in Algorithm 6. Using an argument similar to the one presented for the worst case example in Section 3, we can show that if we use Algorithm 6 where we replace every comparison with an oracle query, the generated clusters can be arbitrarily worse even for small error. In order to improve its robustness, we devise new algorithms to perform assignment of points to respective clusters and farthest point identification. Missing Details from this section are discussed in Appendix 10 and 11.

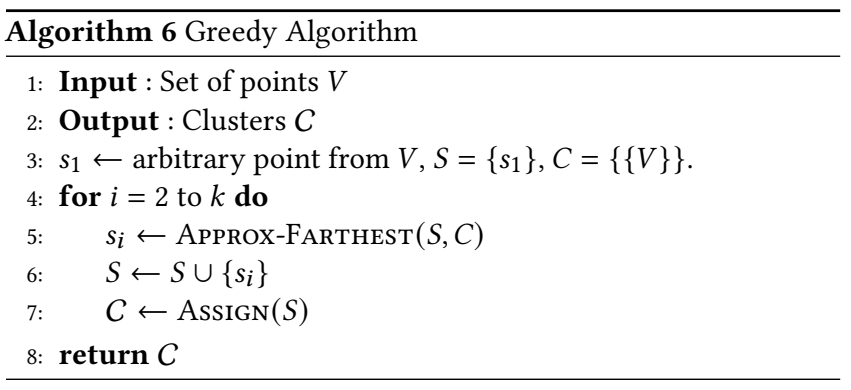

\subsection{Adversarial Noise}

Now, we describe the two steps (Approx-FArThest and Assign) of the Greedy Algorithm that will complete the description of Algorithm 6. To do so, we build upon the results from previous section that give algorithms for obtaining maximum/farthest point.

Approx-Farthest. Given a clustering $C$, and a set of centers $S$, we construct the pairs $\left(v_{i}, s_{j}\right)$ where $v_{i}$ is assigned to cluster $C\left(s_{j}\right)$ centered at $s_{j} \in S$. Using Algorithm 4, we identify the point, center pair that have the maximum distance i.e. $\arg \max _{v_{i} \in V} d\left(v_{i}, s_{j}\right)$, which corresponds to the farthest point. For the parameters, we use $l=\sqrt{n}, t=\log (2 k / \delta)$ and number of samples $\widetilde{V}=\sqrt{n} t$.

Assign. After identifying the farthest point, we reassign all the points to the centers (now including the farthest point as the new center) closest to them. We calculate a movement score called MCount for every point with respect to each center. $\operatorname{MCount}\left(u, s_{j}\right)=$ $\sum_{s_{k} \in S \backslash\left\{s_{j}\right\}} 1\left\{O\left(\left(s_{j}, u\right),\left(s_{k}, u\right)\right)==\right.$ Yes $\}$, for any record $u \in V$ and $s_{j} \in S$. This step is similar to CounT-MAx Algorithm. We assign the point $u$ to the center with the highest MCount value.

EXAMPLE 4.1. Suppose we run $k$-center algorithm with $k=2$ and $\mu=1$ on the points in Example 3.8. The optimal centers are $u$ and $t$ with radius 51 . On running our algorithm, suppose $w$ is chosen as the first center and APPROX-FARTHEST calculates Count values similar to Example 3.2. We have, Count values of $s, t, u, v$ are 1, 2, 3, 0 respectively. Therefore, our algorithm identifies $u$ as the second center, achieving 3-approximation. 
Theoretical Guarantees. We now prove the approximation guarantee obtained by Algorithm 6.

In each iteration, we show that Assign reassigns each point to a center with distance approximately similar to the distance from the closest center. This is surprising given that we only use MCount scores for assignment. Similarly, we show that Approx-FARTHEST (Algorithm 4) identifies a close approximation to the true farthest point. Concretely, we show that every point is assigned to a center which is a $(1+\mu)^{2}$ approximation; Algorithm 4 identifies farthest point $w$ which is a $(1+\mu)^{5}$ approximation.

In every iteration of the Greedy algorithm, if we identify an $\alpha$ approximation of the farthest point, and a $\beta$-approximation when reassigning the points, then, we show that the clusters output are a $2 \alpha \beta^{2}$-approximation to the $k$-center objective. For complete details, please refer Appendix 10. Combining all the claims, for a given error parameter $\mu$, we obtain:

Theorem 4.2. For $\mu<\frac{1}{18}$, Algorithm 6 achieves $a(2+O(\mu))$ approximation for the $k$-center objective using $O\left(n k^{2}+n k \cdot \log ^{2}(k / \delta)\right)$ oracle queries with probability $1-\delta$.

\subsection{Probabilistic Noise}

For probabilistic noise, each query can be incorrect with probability $p$ and therefore, Algorithm 6 may lead to poor approximation guarantees. Here, we build upon the results from section 3.3 and provide Approx-FARThest and Assign algorithms. We denote the size of minimum cluster among optimum clusters $C^{*}$ to be $m$, and total failure probability of our algorithms to be $\delta$. We assume $p \leq 0.40$, a constant strictly less than $\frac{1}{2}$. Let $\gamma=450$ be a large constant used in our algorithms which obtains the claimed guarantees.

Overview. Algorithm 7 presents the pseudo-code of our algorithm that operates in two phases. In the first phase (lines 3-12), we sample each point with a probability $\gamma \log (n / \delta) / m$ to identify a small sample of $\approx \frac{\gamma n \log (n / \delta)}{m}$ points (denoted by $\widetilde{V}$ ) and use Algorithm 7 to identify $k$ centers iteratively. In this process, we also identify a core for each cluster (denoted by $R$ ). Formally, core is defined as a set of $\Theta(\log (n / \delta))$ points that are very close to the center with high probability. The cores are then used in the second phase (line 15) for the assignment of remaining points. Now, we describe

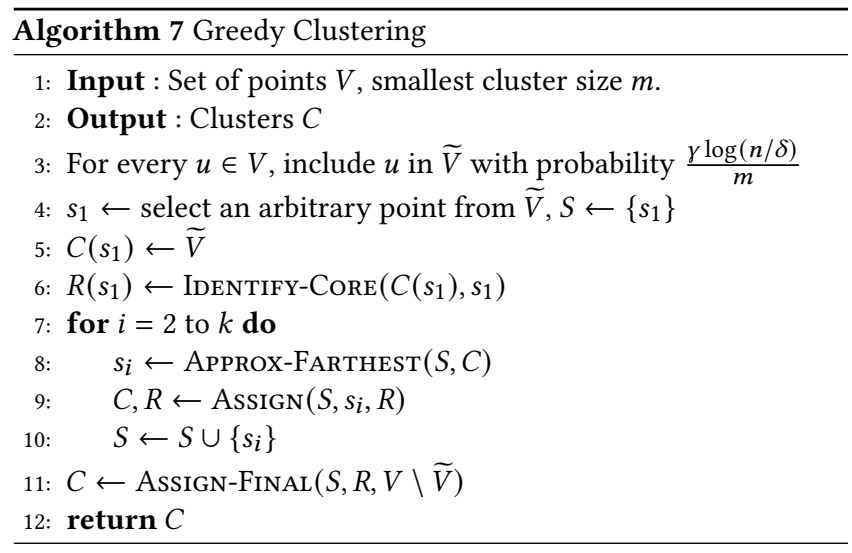

the main challenge in extending Approx-Farthest and Assign ideas of Algorithm 6. Given a cluster $C$ containing the center $s_{i}$, when we find the Approx-Farthest, the ideas from Section 3.2 give a $O\left(\log ^{2} n\right)$ rank approximation. As shown in section 3.3, we can improve the approximation guarantee by considering a set of $\Theta(\log (n / \delta))$ points closest to $s_{i}$, denoted by $R\left(s_{i}\right)$ and call them core of $s_{i}$. We argue that such an assumption of set $R$ is justified. For example, consider the case when clusters are of size $\Theta(n)$ and sampling $k \log (n / \delta)$ points gives us $\log (n / \delta)$ points from each optimum cluster; which means that there $\operatorname{are} \log (n / \delta)$ points within a distance of 2 OPT from every sampled point where OPT refers to the optimum $k$-center objective.

Assign. Consider a point $s_{i}$ such that we have to assign points to form the cluster $C\left(s_{i}\right)$ centered at $s_{i}$. We calculate an assignment score (called ACount in line 4 ) for every point $u$ of a cluster $C\left(s_{j}\right) \backslash$ $R\left(s_{j}\right)$ centered at $s_{j}$. ACount captures the total number of times $u$ is considered to belong to same cluster as that of $x$ for each $x$ in the core $R\left(s_{j}\right)$. Intuitively, points that belong to same cluster as that of $s_{i}$ are expected to have higher ACount score. Based on the scores, we move $u$ to $C\left(s_{i}\right)$ or keep it in $C\left(s_{j}\right)$.
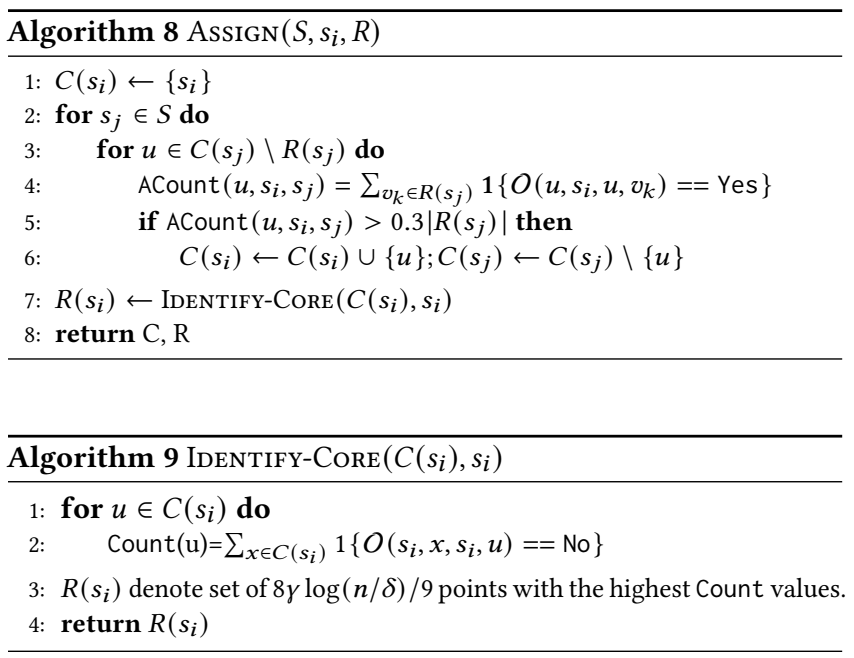

IDENTIFY-CoRE. After forming cluster $C\left(s_{i}\right)$, we identify the core of $s_{i}$. For this, we calculate a score, denoted by Count and captures number of times it is closer to $s_{i}$ compared to other points in $C\left(S_{i}\right)$. Intuitively, we expect points with high values of Count to belong to $C^{*}\left(s_{i}\right)$ i.e., optimum cluster containing $s_{i}$. Therefore we sort these Count scores and return the highest scored points.

Approx-Farthest. For a set of clusters $C$, and a set of centers $S$, we construct the pairs $\left(v_{i}, s_{j}\right)$ where $v_{i}$ is assigned to cluster $C\left(s_{j}\right)$ centered at $s_{j} \in S$ and each center $s_{j} \in S$ has a corresponding core $R\left(s_{j}\right)$. The farthest point can be found by finding the maximum distance (point, center) pair among all the points considered. To do so, we use the ideas developed in section 3.3.

We leverage ClusterComp (Algorithm 10) to compare the distance of two points, say $v_{i}, v_{j}$ from their respective centers $s_{i}, s_{j}$. ClusterComp gives a robust answer to a pairwise comparison query to the oracle $O\left(v_{i}, s_{i}, v_{j}, s_{j}\right)$ using the cores $R\left(s_{i}\right)$ and $R\left(s_{j}\right)$. ClusterComp can be used as a pairwise comparison subroutine in place of PAIRWISECOMP for the algorithm in Section 3 to calculate the farthest point. For every $s_{i} \in S$, let $\widetilde{R}\left(s_{i}\right)$ denote an arbitrary set of $\sqrt{R\left(s_{i}\right)}$ points from $R\left(s_{i}\right)$. For a CLUSTERCOMP comparison 
query between the pairs $\left(v_{i}, s_{i}\right)$ and $\left(v_{j}, s_{j}\right)$, we use these subsets in Algorithm 10 to ensure that we only make $\Theta(\log (n / \delta))$ oracle queries for every comparison. However, when the query is between points of the same cluster, say $C\left(s_{i}\right)$, we use all the $\Theta(\log (n / \delta))$ points from $R\left(s_{i}\right)$. For the parameters used to find maximum using Algorithm 4, we use $l=\sqrt{n}, t=\log (n / \delta)$.

EXAMPLE 4.3. Suppose we run $k$-center Algorithm 7 with $k=2$ and $m=2$ on the points in Example 3.8. Let $w$ denote the first center chosen and Algorithm 7 identifies the core $R(w)$ by calculating Count values. If $O(u, w, s, w)$ and $O(s, w, t, w)$ are answered incorrectly (with probability $p$ ), we obtain Count values of $v, s, u, t$ as 3,2,1,0 respectively; and $v$ is added to $R(w)$. We identify the second center $u$ by calculating FCount for s, $u$ and $t$ (See Fig. 3). After assigning (using AssIGN), the clusters identified are $\{w, v\},\{u, s, t\}$, achieving 3-approximation.

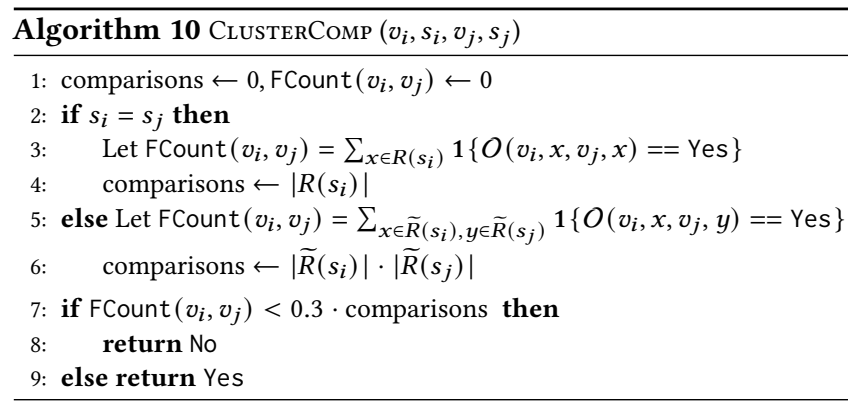

Assign-Final. After obtaining $k$ clusters on the set of sampled points $\widetilde{V}$, we assign the remaining points using ACount scores, similar to the one described in Assign. For every point that is not sampled, we first assign it to $s_{1} \in S$, and if ACount $\left(u, s_{2}, s_{1}\right) \geq$ $0.3\left|R\left(s_{1}\right)\right|$, we re-assign it to $s_{2}$, and continue this process iteratively. After assigning all the points, the clusters are returned as output.

\section{Theoretical Guarantees}

Our algorithm first constructs a sample $\widetilde{V} \subseteq V$ and runs the greedy algorithm on this sampled set of points. Our main idea to ensure that good approximation of the $k$-center objective lies in identifying a good core around each center. Using a sampling probability of $\gamma \log (n / \delta) / m$ ensures that we have at least $\Theta(\log (n / \delta))$ points from each of the optimal clusters in our sampled set $\widetilde{V}$. By finding the closest points using Count scores, we identify $O(\log (n / \delta))$ points around every center that are in the optimal cluster. Essentially, this forms the core of each cluster. These cores are then used for robust pairwise comparison queries (similar to Section 3.3), in our Approx-FARThest and Assign subroutines. We give the following theorem, which guarantees a constant, i.e., $O(1)$ approximation with high probability.

Theorem 4.4. Given $p \leq 0.4$, a failure probability $\delta$, and $m=$ $\Omega\left(\log ^{3}(n / \delta) / \delta\right)$. Then, Algorithm 7 achieves a $O(1)$-approximation for the $k$-center objective using $O\left(n k \log (n / \delta)+\frac{n^{2}}{m^{2}} k \log ^{2}(n / \delta)\right)$ oracle queries with probability $1-O(\delta)$.

\section{HIERARCHICAL CLUSTERING}

In this section, we present robust algorithms for agglomerative hierarchical clustering using single linkage and complete linkage objectives. The naive algorithms initialize every record as a singleton cluster and merge the closest pair of clusters iteratively. For a set of clusters $C=\left\{C_{1}, \ldots, C_{t}\right\}$, the distance between any pair of clusters $C_{i}$ and $C_{j}$, for single linkage clustering, is defined as the minimum distance between any pair of records in the clusters, $d_{S L}\left(C_{1}, C_{2}\right)=\min _{v_{1} \in C_{1}, v_{2} \in C_{2}} d\left(v_{1}, v_{2}\right)$. For complete linkage, cluster distance is defined as the maximum distance between any pair of records. All algorithms discussed in this section can be easily extended for complete linkage, and therefore we study single linkage clustering. The main challenge in implementing single linkage clustering in the presence of adversarial noise is identification of minimum value in a list of at most $\left(\begin{array}{l}n \\ 2\end{array}\right)$ distance values. In each iteration, the closest pair of clusters can be identified by using Algorithm 4 (with $t=2 \log (n / \delta)$ ) to calculate the minimum over the set containing pairwise distances. For this algorithm, Lemma 5.1 shows that the pair of clusters merged in any iteration are a constant approximation of the optimal merge operation at that iteration. The proof of this lemma follows from Theorem 3.6.

LEMMA 5.1. Given a collection of clusters $C=\left\{C_{1}, \ldots, C_{r}\right\}$, our algorithm to calculate the closest pair (using Algorithm 4) identifies $C_{1}$ and $C_{2}$ to merge according to single linkage objective if $d_{S L}\left(C_{2}, C_{2}\right) \leq$ $(1+\mu)^{3} \min _{C_{i}, C_{j} \in C} d\left(C_{i}, C_{j}\right)$ with $1-\delta / n$ probability and requires $O\left(n^{2} \log ^{2}(n / \delta)\right)$ queries.

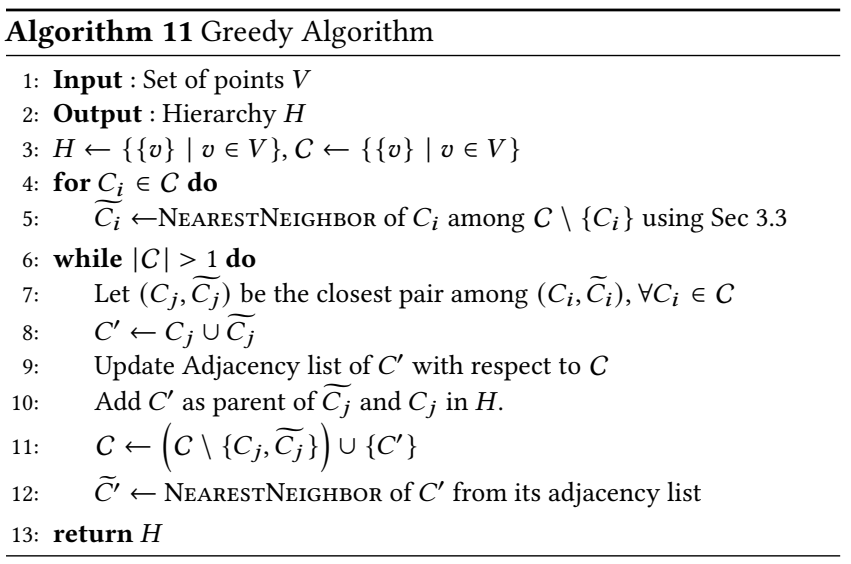

Overview. Agglomerative clustering techniques are known to be inefficient. Each iteration of merge operation compares at most $\left(\begin{array}{l}n \\ 2\end{array}\right)$ pairs of distance values and the algorithm operates $n$ times to construct the hierarchy. This yields an overall query complexity of $O\left(n^{3}\right)$. To improve their query complexity, SLINK algorithm [47] was proposed to construct the hierarchy in $O\left(n^{2}\right)$ comparisons. To implement this algorithm with a comparison oracle, for every cluster $C_{i} \in C$, we maintain an adjacency list containing every cluster $C_{j}$ in $C$ along with a pair of records with the distance equal to the distance between the clusters. For example, the entry for $C_{j}$ in the adjacency list of $C_{i}$ contains the pair of records $\left(v_{i}, v_{j}\right)$ such that $d\left(v_{i}, v_{j}\right)=\min _{v_{i} \in C_{i}, v_{j} \in C_{j}} d\left(v_{i}, v_{j}\right)$. Algorithm 11 presents the pseudo code for single linkage clustering under the adversarial noise model. The algorithm is initialized with singleton clusters where every record is a separate cluster. Then, we identify the closest cluster for every $C_{i} \in \mathcal{C}$, and denote it by $\widetilde{C}_{i}$. This step takes 
$n$ nearest neighbor queries, each requiring $O\left(n \log ^{2}(n / \delta)\right)$ oracle queries. In every subsequent iteration, we identify the closest pair of clusters (Using section 3.3), say $C_{j}$ and $\widetilde{C_{j}}$ from $C$.

After merging these clusters, the data structure is updated as follows. To update the adjacency list, we need the pair of records with minimum distance between the merged cluster $C^{\prime} \equiv$ $C_{j} \cup \widetilde{C_{j}}$ and every other cluster $C_{k} \in C$. In the previous iteration of the algorithm, we already have the minimum distance record pair for $\left(C_{j}, C_{k}\right)$ and $\left(\widetilde{C_{j}}, C_{k}\right)$. Therefore a single query between these two pairs of records is sufficient to identify the minimum distance edge between $C^{\prime}$ and $C_{k}$ (formally: $d_{S L}\left(C_{j} \cup\right.$ $\left.\left.\widetilde{C_{j}}, C_{k}\right)=\min \left\{d_{S L}\left(C_{j}, C_{k}\right), d_{S L}\left(\widetilde{C_{j}}, C_{k}\right)\right\}\right)$. The nearest neighbor of the merged cluster is identified by running minimum calculation over its adjacency list. In Algorithm 11, as we identify closest pair of clusters, each iteration requires $O\left(n \log ^{2}(n / \delta)\right)$ queries. As our Algorithm terminates in at most $n$ iterations, it has an overall query complexity of $O\left(n^{2} \log ^{2}(n / \delta)\right)$. In Theorem 5.2, we given an approximation guarantee for every merge operation of Algorithm 11.

THEOREM 5.2. In any iteration, suppose the distance between a cluster $C_{j} \in C$ and its identified nearest neighbor $\widetilde{C_{j}}$ is $\alpha$-approximation of its distance from the optimal nearest neighbor, then the distance between pair of clusters merged by Algorithm 11 is $\alpha(1+\mu)^{3}$ approximation of the optimal distance between the closest pair of clusters in $C$ with a probability of $1-\delta$ using $O\left(n \log ^{2}(n / \delta)\right)$ oracle queries.

Probabilistic Noise model. The above discussed algorithms do not extend to the probabilistic noise due to constant probability of error for each query. However, when we are given a priori, a partitioning of $V$ into clusters of size $>\log n$ such that the maximum distance between any pair of records in every cluster is smaller than $\alpha$ (a constant), Algorithm 11 can be used to construct the hierarchy correctly. For this case, the algorithm to identify the closest and farthest pair of clusters is same as the one discussed in Section 3.3. Note that agglomerative clustering algorithms are known to require $\Omega\left(n^{2}\right)$ queries, which can be infeasible for million scale datasets. However, blocking based techniques present efficient heuristics to prune out low similarity pairs [44]. Devising provable algorithms with better time complexity is outside the scope of this work.

\section{EXPERIMENTS}

In this section, we evaluate the effectiveness of our techniques on various real world datasets and answer the following questions. Q1: Is quadruplet oracle practically feasible? How do the different types of queries compare in terms of quality and time taken by annotators? Q2: Are proposed techniques robust to different levels of noise in oracle answers? Q3: How does the query complexity and solution quality of proposed techniques compare with optimum for varied levels of noise?

\subsection{Experimental Setup}

Datasets. We consider the following real-world datasets.

(1) cities dataset [2] comprises of $36 \mathrm{~K}$ cities of the United States. The different features of the cities include state, county, zip code, population, time zone, latitude and longitude.

(2) caltech dataset comprises $11.4 \mathrm{~K}$ images from 20 categories. The ground truth distance between records is calculated using the hierarchical categorization as described in [29].

(3) amazon dataset contains $7 \mathrm{~K}$ images and textual descriptions collected from amazon.com [31]. For obtaining the ground truth distances we use Amazon's hierarchical catalog.

(4) monuments dataset comprises of 100 images belonging to 10 tourist locations around the world.

(5) dblp contains $1.8 \mathrm{M}$ titles of computer science papers from different areas [60]. From these titles, noun phrases were extracted and a dictionary of all the phrases was constructed. Euclidean distance in word2vec embedding space is considered as the ground truth distance between concepts.

Baselines. We compare our techniques with the optimal solution (whenever possible) and the following baselines. (a) Tour2 constructs a binary tournament tree over the entire dataset to compare the values and the root node corresponds to the identified maximum/minimum value (Algorithm 2 with $\lambda=2$ ). This approach is an adaptation of the finding maximum algorithm in [15] with a difference that each query is not repeated multiple times to increase success probability. We also use them to identify the farthest and nearest point in the greedy $k$-center Algorithm 6 and closest pair of clusters in hierarchical clustering.

(b) Samp considers a sample of $\sqrt{n}$ records and identifies the farthest/nearest by performing quadratic number of comparisons over the sampled points using CounT-MAx. For $k$-center, Samp considers a sample of $k \log n$ points to identify $k$ centers over these samples using the greedy algorithm. It then assigns all the remaining points to the identified centers by querying each record with every pair of center.

Calculating optimal clustering objective for $k$-center is NP-hard even in the presence of accurate pairwise distance [59]. So, we compare the solution quality with respect to the greedy algorithm on the ground truth distances, denoted by TDist. For farthest, nearest neighbor and hierarchical clustering, TDist denotes the optimal technique that has access to ground truth distance between records.

Our algorithm is labelled Far for farthest identification, NN for nearest neighbor, $\mathrm{kC}$ for $k$-center and $\mathrm{HC}$ for hierarchical clustering with subscript $a$ denoting the adversarial model and $p$ denoting the probabilistic noise model. All algorithms are implemented in $\mathrm{C}++$ and run on a server with 64GB RAM. The reported results are averaged over 100 randomly chosen iterations. Unless specified, we set $t=1$ in Algorithm 4 and $\gamma=2$ in Algorithm 7 .

Evaluation Metric. For finding maximum and nearest neighbors, we compare different techniques by evaluating the true distance of the returned solution from the queried points. For $k$-center, we use the objective value, i.e., maximum radius of the returned clusters as the evaluation metric and compare against the true greedy algorithm (TDist) and other baselines. For datasets where ground truth clusters are known (amazon, caltech and monuments), we use F-score over intra-cluster pairs for comparing it with the baselines [20]. For hierarchical clustering, we compute the pairs of clusters merged in every iteration and compare the average true distance between these clusters. In addition to the quality of returned solution, we compare the query complexity and running time of the proposed techniques with the baselines described above. Noise Estimation. For cities, amazon, caltech, and monuments datasets, we ran a user study on Amazon Mechanical Turk to estimate the noise in oracle answers over a small sample of the dataset, 


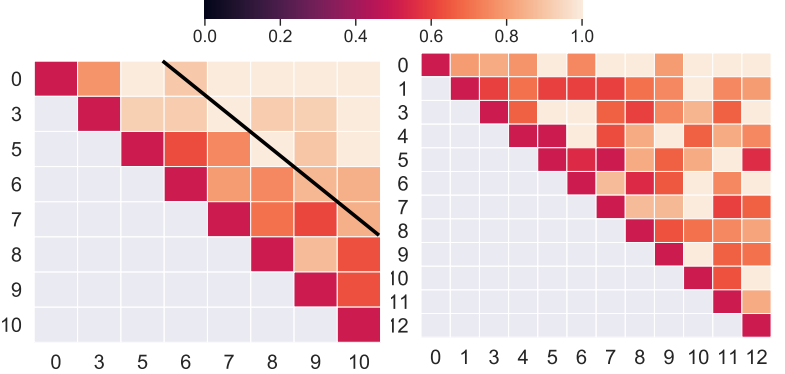

(a) caltech

Figure 4: Accuracy values (denoted by the color of a cell) for different distance ranges observed during our user study. The diagonal entries refer to the quadruplets with similar distance between the corresponding pairs and the distance increases as we go further away from the diagonal.

often referred to as the validation set. Using crowd responses, we trained a classifier (random forest [51] obtained the best results) using active learning to act as the quadruplet oracle, and reduce the number of queries to the crowd. Our active learning algorithm [50] uses a batch of 20 queries and we stop it when the classifier accuracy on the validation set does not improve by more than 0.01 [26] To efficiently construct a small set of candidates for active learning and pruning low similarity pairs for $\mathrm{dblp}$, we employ token based blocking [44] for the datasets. For the synthetic oracle, we simulate quadruplet oracle with different values of the noise parameters.

\subsection{User study}

In this section, we evaluate the users ability to answer quadruplet queries and compare it with other types of queries.

Setup. We ran a user study on Amazon Mechanical Turk platform for four datasets cities, amazon, caltech and monuments. We consider the ground truth distance between record pairs and discretize them into buckets, and assign a pair of records to a bucket if the distance falls within its range. For every pair of buckets, we query a random subset of $\log n$ quadruplet oracle queries (where $n$ is size of dataset). Each query is answered by three different crowd workers and a majority vote is taken as the answer to the query.

6.2.1 Qualitative Analysis of Oracle. In Figure 4, for every pair of buckets, using a heat map, we plot the accuracy of answers obtained from the crowd workers for quadruplet queries. For all datasets, average accuracy of quadruplet queries is more than 0.83 and the accuracy is minimum whenever both pairs of records belong to the same bucket (as low as 0.5 ). However, we observe varied behavior across datasets as the distance between considered pairs increases.

For the caltech dataset, we observe that when the ratio of the distances is more than 1.45 (indicated by a black line in the Figure 4(a)) , there is no noise (or close to zero noise) observed in the query responses. As we observe a sharp decline in noise as the distance between the pairs increases, it suggests that adversarial noise is satisfied for this dataset. We observe a similar pattern for the cities and monuments datasets. For the amazon dataset, we observe that there is substantial noise across all distance ranges (See Figure 4(b)) rather than a sharp decline, suggesting that the probabilistic model is satisfied.

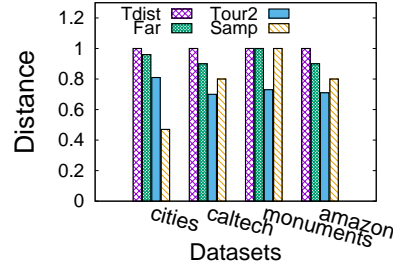

(a) Farthest,higher is better

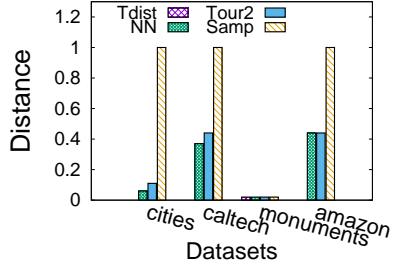

(b) Nearest Neighbor (NN), lower is better
Figure 5: Comparison of farthest and NN techniques for crowdsourced oracle queries.

6.2.2 Comparison with pairwise querying mechanisms. To evaluate the benefit of quadruplet queries, we compare the quality of quadruplet comparison oracle answers with the following pairwise oracle query models. (a) Optimal cluster query: This query asks questions of type 'do $u$ and $v$ refer to same/similar type?'. (b) Distance query: How similar are the records $x$ and $y$ ? In this query, the annotator scores the similarity of the pair within 1 to 10 .

We make the following observations. (i) Optimal cluster queries are answered correctly only if the ground truth clusters refer to different entities (each cluster referring to a distinct entity). Crowd workers tend to answer 'No' if the pair of records refer to different entities. Therefore, we observe high precision (more than 0.90 ) but low recall $(0.50$ on amazon and 0.30 on caltech for $k=10)$ of the returned labels. (ii) We observed very high variance in the distance estimation query responses. For all record pairs with identical entities, the users returned distance estimates that were within $20 \%$ of the correct distances. In all other cases, we observe the estimates to have errors of upto $50 \%$. We provide more detailed comparison on the quality of clusters identified by pairwise query responses along with quadruplet queries in the next section.

\subsection{Crowd Oracle: Solution Quality \& Query Complexity}

In this section, we compare the quality of our proposed techniques for the datasets on which we performed the user study. Following the findings of Section 6.2, we use probabilistic model based algorithm for amazon (with $p=0.50$ ) and adversarial noise model based algorithm for caltech, monuments and cities.

Finding Max and Farthest/Nearest Neighbor. Figure 5 compares the quality of farthest and nearest neighbor (NN) identified by proposed techniques along with other baselines. The values are normalized according to the maximum value to present all datasets on the same scale. Across all datasets, the point identified by Far and $\mathrm{NN}$ is closest to the optimal value, TDist. In contrast, the farthest returned by Tour2 is better than that of Samp for cities dataset but not for caltech, monuments and amazon. We found that this difference in quality across datasets is due to varied distance distribution between pairs. The cities dataset has a skewed distribution of distance between record pairs, leading to a unique optimal solution to the farthest/NN problem. Due to this reason, the set of records sampled by Samp does not contain any record that is a good approximation of the optimal farthest. However, ground truth distances between record pairs in amazon, monuments and caltech are less skewed with more than $\log n$ records satisfying the optimal farthest point for all queries. Therefore, Samp performs better than Tour2 


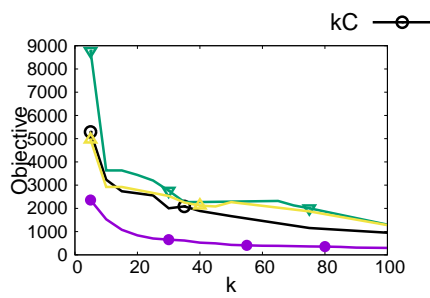

(a) cities, $\mu=1$

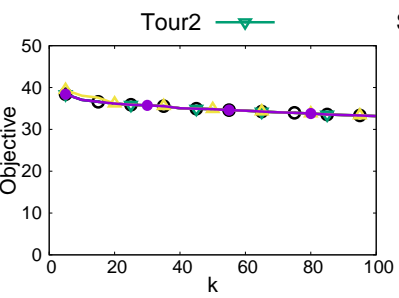

(d) dblp, $\mu=0.5$

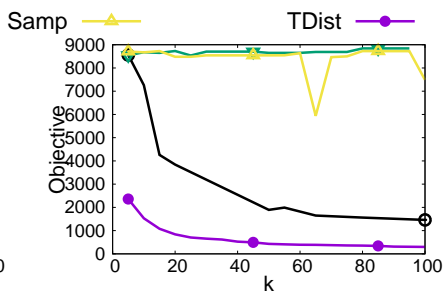

(c) cities, $\mathrm{p}=0.1$

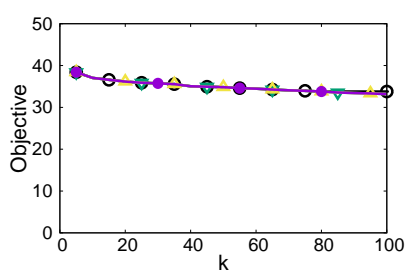

(d) dblp, $p=0.1$

Figure 6: $k$-center clustering objective comparison for adversarial and probabilistic noise model.

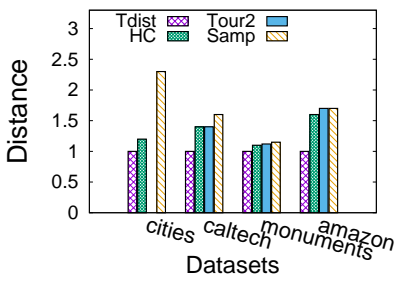

(a) Single Linkage

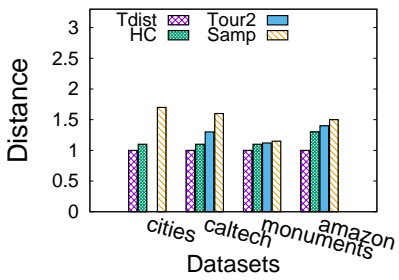

(b) Complete Linkage
Figure 7: Comparison of Hierarchical clustering techniques with crowdsourced oracle.

on these datasets. We observe Samp performs worse for NN because our sample does not always contain the closest point.

$k$-center Clustering. We evaluate the F-score ${ }^{2}$ of the clusters generated by our techniques along with baselines and techniques for pairwise optimal query mechanism (denoted as $0 \mathrm{q})^{3}$. Table 1 presents the summary of our results for different values of $k$. Across all datasets, our technique achieves more than $0.90 \mathrm{~F}$-score. On the other hand, Tour 2 and Samp do not identify the ground truth clusters correctly, leading to low F-score. Similarly, Oq achieves poor recall (and hence low F-score) as it labels many record pairs to belong to separate clusters. For example, a frog and a butterfly belong to the same optimal cluster for caltech $(k=10)$ but the two records are assigned to different clusters by $0 q$.

Hierarchical Clustering. Figure 7 compares the average distance of the merged clusters across different iterations of the agglomerative clustering algorithm. Tour 2 has $O\left(n^{3}\right)$ complexity and does not run for cities dataset in less than $48 \mathrm{hrs}$. The objective value of different techniques are normalized by the optimal value with Tdist denoting 1. For all datasets, HC performs better than Samp and Tour2. Among datasets, the quality of hierarchies generated for monuments is similar for all techniques due to low noise.

Query Complexity. To ensure scalability, we trained active learning based classifier for all the aforementioned experiments. In total, amazon, cities, and caltech required 540 (cost: \$32.40), 220 (cost: $\$ 13.20$ ) and 280 (cost: \$16.80) queries to the crowd respectively.

\subsection{Simulated Oracle: Solution Quality \& Query Complexity}

In this section, we compare the robustness of the techniques where the query response is simulated synthetically for given $\mu$ and $p$.

\footnotetext{
${ }^{2}$ Optimal clusters are identified from the original source of the datasets (amazon and caltech) and manually for monuments.

${ }^{3}$ We report the results on the sample of queries asked to the crowd as opposed to training a classifier because the classifier generates noisier results and has poorer F-score than the quality of labels generated by crowdsourcing
}

\begin{tabular}{|c|c|c|c|c|}
\hline Technique & $\mathrm{kC}$ & Tour2 & Samp & $0^{*}$ \\
\hline caltech $(k=10)$ & $\mathbf{1}$ & 0.88 & 0.91 & 0.45 \\
caltech $(k=15)$ & $\mathbf{1}$ & 0.89 & 0.88 & 0.49 \\
caltech $(k=20)$ & $\mathbf{0 . 9 9}$ & 0.93 & 0.87 & 0.58 \\
\hline monuments $(k=5)$ & $\mathbf{1}$ & 0.95 & 0.97 & 0.77 \\
\hline amazon $(k=7)$ & $\mathbf{0 . 9 6}$ & 0.74 & 0.57 & 0.48 \\
amazon $(k=14)$ & $\mathbf{0 . 9 2}$ & 0.66 & 0.54 & 0.72 \\
\hline
\end{tabular}

Table 1: F-score comparison of $k$-center clustering. $0 q$ is marked with $*$ as it was computed on a sample of 150 pairwise queries to the crowd ${ }^{3}$. All other techniques were run on the complete dataset using a classifier.

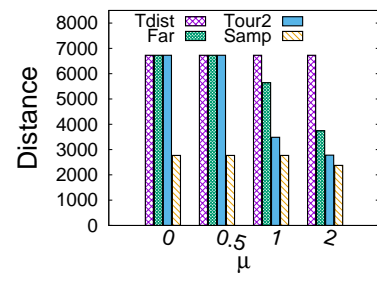

(a) cities-Adversarial

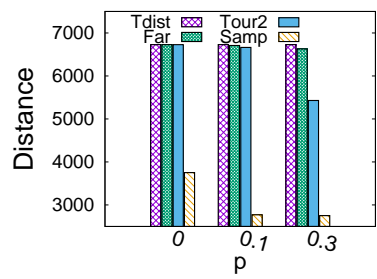

(b) cities-Probabilistic
Figure 8: Comparison of farthest identification techniques for adversarial and probabilistic noise models.

Finding Max and Farthest/Nearest Neighbor. In Figure 8(a), $\mu=0$ denotes the setting where the oracle answers all queries correctly. In this case, Far and Tour 2 identify the optimal solution but Samp does not identify the optimal solution for cities. In both datasets, Far identifies the correct farthest point for $\mu<1$. Even with an increase in noise $(\mu)$, we observe that the farthest is always at a distance within 4 times the optimal distance (See Fig 8(a)). We observe that the quality of farthest identified by Tour2 is close to that of Far for smaller $\mu$ because the optimal farthest point $v_{\max }$ has only a few points in the confusion region $C$ (See Section 3) that contains the points that are close to $v_{\max }$. For e.g., less than $10 \%$ are present in $C$ when $\mu=1$ for cities dataset, i.e., less than $10 \%$ points return erroneous answer when compared with $v_{\max }$.

In Figure 8(b), we compare the true distance of the identified farthest points for the case of probabilistic noise with error probability $p$. We observe that $\operatorname{Far}_{p}$ identifies points with distance values very close to the farthest distance Tdist, across all data sets and error values. This shows that Far performs significantly better than the theoretical approximation presented in Section 3. On the other hand, the solution returned by Samp is more than $4 \times$ smaller than the value returned by $\operatorname{Far}_{p}$ for an error probability of 0.3 . Tour 2 has a similar performance as that of $\operatorname{Far}_{p}$ for $p \leq 0.1$, but we observe a decline in solution quality for higher noise $(p)$ values. 


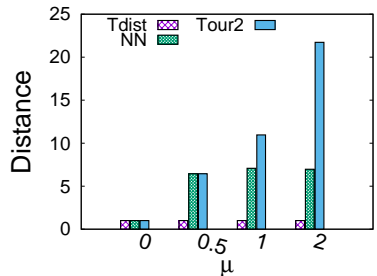

(a) cities-Adversarial

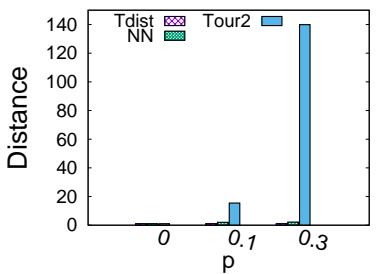

(b) cities-Probabilistic
Figure 9: Comparison of nearest neighbor techniques for adversarial and probabilistic noise model (lower is better).

In Figures 9(a), 9(b), we compare the true distance of the identified nearest neighbor with different baselines.

NN shows superior performance as compared to Tour 2 across all error values. This justifies the lack of robustness of Tour2 as discussed in Section 3. The solution quality of NN does not worsen with increase in error. We omit Samp from the plots because the returned points had very poor performance (as bad as 700 even in the absence of error). We observed similar behavior for other datasets. In terms of query complexity, NN requires around $53 \times 10^{3}$ queries for cities dataset and the number of queries grow linearly with the dataset size. Among baselines, Tour 2 uses $37 \times 10^{3}$ queries and Samp uses $18 \times 10^{3}$.

"In conclusion, we observe that our techniques achieve the best quality across all data sets and error values, while Tour2 performs similar to Far for low error, and its quality degrades with increasing error."

$k$-center Clustering. Figure 6 compares the $k$-center objective of the returned clusters for varying $k$ in the adversarial and probabilistic noise model. Tdist denotes the best possible clustering objective, which is guaranteed to be a 2-approximation of the optimal objective. The set of clusters returned by $\mathrm{kC}$ are consistently very close to TDist across all datasets, validating the theory. For higher values of $k, \mathrm{kC}$ approaches closer to TDist, thereby improving the approximation guarantees. The quality of clusters identified by $\mathrm{kC}$ are similar to that of Tour2 and Far for adversarial noise (Figure 6a,b) but considerably better for probabilistic noise (Figure 6c,d).

Running time. Table 2 compares the running time and the number of required quadruplet comparisons for various problems under adversarial noise model with $\mu=1$ for the largest $\mathrm{dblp}$ dataset. Far and NN requires less than 6 seconds for both adversarial and probabilistic error models. Our $k$-center clustering technique requires less than $450 \mathrm{~min}$ to identify 50 centers for dblp dataset across different noise models; the running time grows linearly with $k$. While the running time of our algorithms are slightly higher than Tour 2 for farthest, nearest and $k$-center, Tour 2 did not finish in $48 \mathrm{hrs}$ due to $O\left(n^{3}\right)$ running time for single and complete linkage hierarchical clustering. We observe similar performance for the probabilistic noise model. Note that even though the number of comparisons are in millions, this dataset requires only 740 queries to the crowd workers to train the classifier.

\section{CONCLUSION}

In this paper, we show how algorithms for various basic tasks such as finding maximum, nearest neighbor, $k$-center clustering, and agglomerative hierarchical clustering can be designed using distance based comparison oracle in presence of noise. We believe

\begin{tabular}{|c|c|c|c|c|c|c|}
\hline Problem & \multicolumn{2}{|c|}{ Our Approach } & \multicolumn{2}{|c|}{ Tour2 } & \multicolumn{2}{|c|}{ Samp } \\
\hline & Time & \# Comp & Time & \# Comp & Time & \# Comp \\
\hline Farthest & 0.1 & $2.2 \mathrm{M}$ & 0.06 & $2 \mathrm{M}$ & 0.07 & $1 \mathrm{M}$ \\
\hline Nearest & 0.075 & $2 \mathrm{M}$ & 0.07 & $2 \mathrm{M}$ & 0.61 & $1 \mathrm{M}$ \\
\hline kC (k=50) & 450 & $120 \mathrm{M}$ & 375.3 & $95 \mathrm{M}$ & 477 & $105 \mathrm{M}$ \\
\hline Single Linkage & 1813 & $990 \mathrm{M}$ & \multicolumn{2}{|c|}{ DNF } & 1760 & $940 \mathrm{M}$ \\
\hline Complete Linkage & 1950 & $940 \mathrm{M}$ & \multicolumn{2}{|c|}{ DNF } & 1940 & $920 \mathrm{M}$ \\
\hline
\end{tabular}

Table 2: Running time (in minutes) and number of quadruplet comparisons (denoted by \# Comp, in millions) of different techniques for $\mathrm{dblp}$ dataset under the adversarial noise model with $\mu=1$. DNF denotes 'did not finish'.

our techniques can be useful for other clustering tasks such as $k$-means and $k$-median, and we leave those as future work.

\section{REFERENCES}

[1] Google vision api https://cloud.google.com/vision.

[2] United states cities database. https://simplemaps.com/data/us-cities.

[3] Nir Ailon, Anup Bhattacharya, Ragesh Jaiswal, and Amit Kumar. Approximate clustering with same-cluster queries. In 9th Innovations in Theoretical Computer Science Conference (ITCS 2018), volume 94, page 40. Schloss Dagstuhl-LeibnizZentrum fuer Informatik, 2018.

[4] Miklós Ajtai, Vitaly Feldman, Avinatan Hassidim, and Jelani Nelson. Sorting and selection with imprecise comparisons. In International Colloquium on Automata, Languages, and Programming, pages 37-48. Springer, 2009.

[5] Akhil Arora, Sakshi Sinha, Piyush Kumar, and Arnab Bhattacharya. Hd-index: pushing the scalability-accuracy boundary for approximate knn search in highdimensional spaces. Proceedings of the VLDB Endowment, 11(8):906-919, 2018.

[6] Hassan Ashtiani, Shrinu Kushagra, and Shai Ben-David. Clustering with samecluster queries. In Advances in neural information processing systems, pages 3216-3224, 2016.

[7] Shai Ben-David. Clustering-what both theoreticians and practitioners are doing wrong. In Thirty-Second AAAI Conference on Artificial Intelligence, 2018.

[8] Mark Braverman, Jieming Mao, and S Matthew Weinberg. Parallel algorithms for select and partition with noisy comparisons. In Proceedings of the forty-eighth annual ACM symposium on Theory of Computing, pages 851-862, 2016.

[9] Mark Braverman and Elchanan Mossel. Noisy sorting without resampling. In Proceedings of the nineteenth annual ACM-SIAM symposium on Discrete algorithms, pages 268-276. Society for Industrial and Applied Mathematics, 2008.

[10] Marco Bressan, Nicolò Cesa-Bianchi, Andrea Paudice, and Fabio Vitale. Correlation clustering with adaptive similarity queries. In Advances in Neural Information Processing Systems, pages 12510-12519, 2019.

[11] Vaggos Chatziafratis, Rad Niazadeh, and Moses Charikar. Hierarchical clustering with structural constraints. arXiv preprint arXiv:1805.09476, 2018.

[12] I Chien, Chao Pan, and Olgica Milenkovic. Query k-means clustering and the double dixie cup problem. In Advances in Neural Information Processing Systems, pages $6649-6658,2018$.

[13] Tuhinangshu Choudhury, Dhruti Shah, and Nikhil Karamchandani. Top-m clustering with a noisy oracle. In 2019 National Conference on Communications (NCC), pages 1-6. IEEE, 2019.

[14] Eleonora Ciceri, Piero Fraternali, Davide Martinenghi, and Marco Tagliasacchi. Crowdsourcing for top-k query processing over uncertain data. IEEE Transactions on Knowledge and Data Engineering, 28(1):41-53, 2015

[15] Susan Davidson, Sanjeev Khanna, Tova Milo, and Sudeepa Roy. Top-k and clustering with noisy comparisons. ACM Trans. Database Syst., 39(4), December 2015.

[16] Eyal Dushkin and Tova Milo. Top-k sorting under partial order information. In Proceedings of the 2018 International Conference on Management of Data, pages 1007-1019, 2018.

[17] Ehsan Emamjomeh-Zadeh and David Kempe. Adaptive hierarchical clustering using ordinal queries. In Proceedings of the Twenty-Ninth Annual ACM-SIAM Symposium on Discrete Algorithms, pages 415-429. SIAM, 2018.

[18] Uriel Feige, Prabhakar Raghavan, David Peleg, and Eli Upfal. Computing with noisy information. SIAM Journal on Computing, 23(5):1001-1018, 1994.

[19] Donatella Firmani, Barna Saha, and Divesh Srivastava. Online entity resolution using an oracle. PVLDB, 9(5):384-395, 2016.

[20] Sainyam Galhotra, Donatella Firmani, Barna Saha, and Divesh Srivastava. Robust entity resolution using random graphs. In Proceedings of the 2018 International Conference on Management of Data, pages 3-18, 2018.

[21] Barbara Geissmann, Stefano Leucci, Chih-Hung Liu, and Paolo Penna. Sorting with recurrent comparison errors. In 28th International Symposium on Algorithms and Computation (ISAAC 2017). Schloss Dagstuhl-Leibniz-Zentrum fuer Informatik, 2017.

[22] Barbara Geissmann, Stefano Leucci, Chih-Hung Liu, and Paolo Penna. Optimal sorting with persistent comparison errors. In 27th Annual European Symposium on 
Algorithms (ESA 2019), volume 144, page 49. Schloss Dagstuhl-Leibniz-Zentrum für Informatik, 2019.

[23] Barbara Geissmann, Stefano Leucci, Chih-Hung Liu, and Paolo Penna. Optimal dislocation with persistent errors in subquadratic time. Theory of Computing Systems, 64(3):508-521, 2020.

[24] Debarghya Ghoshdastidar, Michaël Perrot, and Ulrike von Luxburg. Foundations of comparison-based hierarchical clustering. In Advances in Neural Information Processing Systems, pages 7454-7464, 2019.

[25] Yogesh Girdhar and Gregory Dudek. Efficient on-line data summarization using extremum summaries. In 2012 IEEE International Conference on Robotics and Automation, pages 3490-3496. IEEE, 2012.

[26] Chaitanya Gokhale, Sanjib Das, AnHai Doan, Jeffrey F Naughton, Narasimhan Rampalli, Jude Shavlik, and Xiaojin Zhu. Corleone: Hands-off crowdsourcing for entity matching. In Proceedings of the 2014 ACM SIGMOD international conference on Management of data, pages 601-612, 2014.

[27] Teofilo F Gonzalez. Clustering to minimize the maximum intercluster distance. Theoretical Computer Science, 38:293-306, 1985.

[28] Kasper Green Larsen, Michael Mitzenmacher, and Charalampos Tsourakakis. Clustering with a faulty oracle. In Proceedings of The Web Conference 2020, WWW '20, page 2831-2834, New York, NY, USA, 2020. Association for Computing Machinery.

[29] Gregory Griffin, Alex Holub, and Pietro Perona. Caltech-256 object category dataset. 2007.

[30] Stephen Guo, Aditya Parameswaran, and Hector Garcia-Molina. So who won? dynamic max discovery with the crowd. In Proceedings of the 2012 ACM SIGMOD International Conference on Management of Data, pages 385-396, 2012.

[31] Ruining He and Julian McAuley. Ups and downs: Modeling the visual evolution of fashion trends with one-class collaborative filtering. In proceedings of the 25th international conference on world wide web, pages 507-517, 2016.

[32] Max Hopkins, Daniel Kane, Shachar Lovett, and Gaurav Mahajan. Noisetolerant, reliable active classification with comparison queries. arXiv preprint arXiv:2001.05497, 2020

[33] Wasim Huleihel, Arya Mazumdar, Muriel Médard, and Soumyabrata Pal. Samecluster querying for overlapping clusters. In Advances in Neural Information Processing Systems, pages 10485-10495, 2019.

[34] Christina Ilvento. Metric learning for individual fairness. arXiv preprint arXiv:1906.00250, 2019

[35] Ehsan Kazemi, Lin Chen, Sanjoy Dasgupta, and Amin Karbasi. Comparison based learning from weak oracles. arXiv preprint arXiv:1802.06942, 2018.

[36] Taewan Kim and Joydeep Ghosh. Relaxed oracles for semi-supervised clustering. arXiv preprint arXiv:1711.07433, 2017.

[37] Taewan Kim and Joydeep Ghosh. Semi-supervised active clustering with weak oracles. arXiv preprint arXiv:1709.03202, 2017

[38] Rolf Klein, Rainer Penninger, Christian Sohler, and David P Woodruff. Tolerant algorithms. In European Symposium on Algorithms, pages 736-747. Springer, 2011.

[39] Matthäus Kleindessner, Pranjal Awasthi, and Jamie Morgenstern. Fair k-center clustering for data summarization. In International Conference on Machine Learning, pages 3448-3457, 2019.

[40] Ngai Meng Kou, Yan Li, Hao Wang, Leong Hou U, and Zhiguo Gong. Crowdsourced top-k queries by confidence-aware pairwise judgments. In Proceedings of the 2017 ACM International Conference on Management of Data, pages 1415-1430, 2017

[41] Blake Mason, Ardhendu Tripathy, and Robert Nowak. Learning nearest neighbor graphs from noisy distance samples. In Advances in Neural Information Processing Systems, pages 9586-9596, 2019.

[42] Arya Mazumdar and Barna Saha. Clustering with noisy queries. In Advances in Neural Information Processing Systems, pages 5788-5799, 2017.

[43] Arya Mazumdar and Barna Saha. Query complexity of clustering with side information. In Advances in Neural Information Processing Systems, pages 46824693, 2017.

[44] George Papadakis, Jonathan Svirsky, Avigdor Gal, and Themis Palpanas. Comparative analysis of approximate blocking techniques for entity resolution. Proceedings of the VLDB Endowment, 9(9):684-695, 2016.

[45] Vassilis Polychronopoulos, Luca De Alfaro, James Davis, Hector Garcia-Molina, and Neoklis Polyzotis. Human-powered top-k lists. In WebDB, pages 25-30, 2013.

[46] Dražen Prelec, H Sebastian Seung, and John McCoy. A solution to the singlequestion crowd wisdom problem. Nature, 541(7638):532-535, 2017

[47] Robin Sibson. Slink: an optimally efficient algorithm for the single-link cluster method. The computer journal, 16(1):30-34, 1973.

[48] Omer Tamuz, Ce Liu, Serge Belongie, Ohad Shamir, and Adam Tauman Kalai Adaptively learning the crowd kernel. In Proceedings of the 28th International Conference on International Conference on Machine Learning, pages 673-680, 2011.

[49] Antti Ukkonen. Crowdsourced correlation clustering with relative distance comparisons. In 2017 IEEE International Conference on Data Mining (ICDM), pages 1117-1122. IEEE, 2017.

[50] https://modal-python.readthedocs.io/en/latest/. modal library.

[51] https://scikit-learn.org/stable/. Scikit-learn.
[52] Vijay V Vazirani. Approximation algorithms. Springer Science \& Business Media, 2013.

[53] Petros Venetis, Hector Garcia-Molina, Kerui Huang, and Neoklis Polyzotis. Max algorithms in crowdsourcing environments. In Proceedings of the 21st international conference on World Wide Web, pages 989-998, 2012.

[54] Victor Verdugo. Skyline computation with noisy comparisons. In Combinatorial Algorithms: 31st International Workshop, IWOCA 2020, Bordeaux, France, June 8-10, 2020, Proceedings, page 289. Springer.

[55] Vasilis Verroios and Hector Garcia-Molina. Entity resolution with crowd errors. In 2015 IEEE 31st International Conference on Data Engineering, pages 219-230. IEEE, 2015.

[56] Norases Vesdapunt, Kedar Bellare, and Nilesh Dalvi. Crowdsourcing algorithms for entity resolution. Proceedings of the VLDB Endowment, 7(12):1071-1082, 2014.

[57] Ramya Korlakai Vinayak and Babak Hassibi. Crowdsourced clustering: Querying edges vs triangles. In Advances in Neural Information Processing Systems, pages 1316-1324, 2016.

[58] Jiannan Wang, Tim Kraska, Michael J Franklin, and Jianhua Feng. Crowder: Crowdsourcing entity resolution. Proceedings of the VLDB Endowment, 5(11), 2012.

[59] David P Williamson and David B Shmoys. The design of approximation algorithms. Cambridge university press, 2011.

[60] Chao Zhang, Fangbo Tao, Xiusi Chen, Jiaming Shen, Meng Jiang, Brian Sadler, Michelle Vanni, and Jiawei Han. Taxogen: Unsupervised topic taxonomy construction by adaptive term embedding and clustering. In Proceedings of the 24th ACM SIGKDD International Conference on Knowledge Discovery \& Data Mining, pages 2701-2709, 2018. 


\section{FINDING MAXIMUM}

Lemma 8.1. (Hoeffding's Inequality) If $X_{1}, X_{2}, \cdots, X_{n}$ are independent random variables with $a_{i} \leq X_{i} \leq b_{i}$ for all $i \in[n]$, then

$$
\operatorname{Pr}\left[\left|\sum_{i} X_{i}-\mathbf{E}\left[X_{i}\right]\right| \geq n \epsilon\right] \leq 2 \exp \left(-\frac{2 n^{2} \epsilon^{2}}{\sum_{i}\left(b_{i}-a_{i}\right)^{2}}\right)
$$

\subsection{Adversarial Noise}

Let the maximum value among $V$ be denoted by $v_{\max }$ and the set of records for which the oracle answer can be incorrect is given by

$$
C=\left\{u \mid u \in V, u \geq \frac{v_{\max }}{1+\mu}\right\}
$$

Claim 8.2. For any partition $V_{i}$, Tournament $\left(V_{i}\right)$ uses at most $2\left|V_{i}\right|$ oracle queries.

Proof. Consider the $i$ th round in Tournament. We can observe that the number of remaining values is at most $\frac{\left|V_{i}\right|}{2^{i}}$. So, we make $\frac{\left|V_{i}\right|}{2^{i+1}}$ many oracle queries in this round. Total number of oracle queries made is

$$
\sum_{i=0}^{\log n} \frac{\left|V_{i}\right|}{2^{i+1}} \leq 2\left|V_{i}\right|
$$

Lemma 8.3. Given a set of values $S$, Count-MAX $(S)$ returns a $(1+\mu)^{2}$ approximation of maximum value of $S$ using $O\left(|S|^{2}\right)$ oracle queries.

Proof. Let $v_{\max }=\max \{x \in S\}$. Consider a value $w \in S$ such that $w<\frac{v_{\max }}{(1+\mu)^{2}}$. We compare the Count values for $v_{\max }$ and $w$ given by, Count $\left(v_{\max }, S\right)=\sum_{x \in S} 1\left\{O\left(v_{\max }, x\right)==\right.$ No $\}$ and Count $(w, S)=\sum_{x \in S} 1\{O(w, x)==$ No $\}$. We argue that $w$ can never be returned by Algorithm 1, i.e., $\operatorname{Count}(w, S)<\operatorname{Count}\left(v_{\max }, S\right)$.

$$
\begin{aligned}
\operatorname{Count}\left(v_{\max }, S\right)=\sum_{x \in S} 1\left\{O\left(v_{\max }, x\right)==\text { No }\right\} & \geq \sum_{x \in S \backslash\left\{v_{\max }\right\}} 1\left\{x<v_{\max } /(1+\mu)\right\} \\
& =1\left\{O\left(v_{\max }, w\right)==\text { No }\right\}+\sum_{x \in S \backslash\left\{v_{\max }, w\right\}} 1\left\{x<v_{\max } /(1+\mu)\right\} \\
& =1+\sum_{x \in S \backslash\left\{v_{\max }, w\right\}} 1\left\{x<v_{\max } /(1+\mu)\right\} \\
\operatorname{Count}(w, S)=\sum_{y \in S} 1\{O(w, y)==\text { No }\} & =\sum_{y \in S \backslash\left\{w, v_{\max }\right\}} 1\{O(w, y)==\text { No }\} \\
& \leq \sum_{y \in S \backslash\left\{w, v_{\max }\right\}} 1\{y \leq(1+\mu) w\} \\
& \leq \sum_{y \in S \backslash\left\{w, v_{\max }\right\}} 1\left\{y \leq v_{\max } /(1+\mu)\right\}
\end{aligned}
$$

Combining the two, we have :

$$
\operatorname{Count}\left(v_{\max }, S\right)>\operatorname{Count}(w, S)
$$

This shows that the Countof $v_{\max }$ is strictly greater than the count of any point $w$ with $w<\frac{v_{\max }}{(1+\mu)^{2}}$. Therefore, our algorithm would have output $v_{\max }$ instead of $w$. For calculating the Count for all values in $S$, we make at most $|S|^{2}$ oracle queries as we compare every value with every other value. Finally, we output the maximum value as the value with highest Count. Hence, the claim.

Lemma 8.4 (Lemma 3.3 Restated). Suppose $v_{\text {max }}$ is the maximum value among the set of records $V$. Algorithm 2 outputs a value $u_{\text {max }}$ such that $u_{\max } \geq \frac{v_{\max }}{(1+\mu)^{2 \log _{\lambda} n}}$ using $O(n \lambda)$ oracle queries.

Proof. From Lemma 8.3, we have that we lose a factor of $(1+\mu)^{2}$ in each level of the tournament tree, we have that after $\log _{\lambda} n$ levels, the final output will have an approximation guarantee of $(1+\mu)^{2 \log _{\lambda} n}$. The total number of queries used is given by : $\sum_{i=0}^{\log _{\lambda} n} \frac{\left|V_{i}\right|}{\lambda} \lambda^{2}=O(n \lambda)$ where $V_{i}$ is the number of records at level $i$.

Lemma 8.5. Suppose $|C|>\sqrt{n} / 2$. Let $\widetilde{V}$ denote a set of $2 \sqrt{n} \log (2 / \delta)$ samples obtained by uniform sampling with replacement from $V$. Then, $\widetilde{V}$ contains $a(1+\mu)$ approximation of the maximum value $v_{\text {max }}$, with probability $1-\delta / 2$. 
Proof. Consider the first step where we use a uniformly random sample $\widetilde{V}$ of $\sqrt{n} t=2 \sqrt{n} \log (2 / \delta)$ values from $V$ (obtained by sampling with replacement). Given $|C| \geq \frac{\sqrt{n}}{2}$, probability that $\widetilde{V}$ contains a value from $C$ is given by

$$
\operatorname{Pr}[\widetilde{V} \cap C \neq \phi]=1-\left(1-\frac{|C|}{n}\right)^{|\widetilde{V}|}>1-\left(1-\frac{1}{2 \sqrt{n}}\right)^{2 \sqrt{n} \log (2 / \delta)}>1-\delta / 2
$$

So, with probability $1-\delta / 2$, there exists a value $u \in C \cap \widetilde{V}$. Hence, the claim.

LEMma 8.6. Suppose the partition $V_{i}$ contains the maximum value $v_{\max }$ of $V$. If $|C| \leq \sqrt{n} / 2$, then, TOURNAMENT( $\left.V_{i}\right)$ returns the $v_{\text {max }}$ with probability $1 / 2$.

Proof. Algorithm 4 uses a modified tournament tree that partitions the set $V$ into $l=\sqrt{n}$ parts of size $\frac{n}{l}=\sqrt{n}$ each and identifies a maximum $p_{i}$ from each partition $V_{i}$ using Algorithm 2. If $v_{\max } \in V_{i}$, then,

$$
\mathrm{E}\left[\left|C \cap V_{i}\right|\right]=\frac{|C|}{l}=\frac{\sqrt{n}}{2 \sqrt{n}}=\frac{1}{2}
$$

Using Markov's inequality, the probability that $V_{i}$ contains a value from $C$ is given by :

$$
\operatorname{Pr}\left[\left|C \cap V_{i}\right| \geq 1\right] \leq \mathrm{E}\left[\left|C \cap V_{i}\right|\right] \leq \frac{1}{2}
$$

Therefore, with at least a probability of $\frac{1}{2}$, $v_{\max }$ will never be compared with any point from $C$ in the partition $V_{i}$ containing $v_{\max }$. Hence, $v_{\max }$ is returned by Tournament $\left(V_{i}\right)$ with probability $1 / 2$.

LEMMA 8.7 (LEMMA 3.5 RESTATED). $1-\delta / 2$

(2) Suppose $|C| \leq \sqrt{n} / 2$. Then, $T$ contains $v_{\max }$ with a probability at least $1-\delta / 2$.

Proof. Claim (1) follows from Lemma 8.5.

In every iteration $i \leq t$ of Algorithm 4, we have that $v_{\max } \in T_{i}$ with probability $\frac{1}{2}$ (Using Lemma 8.6). To increase the success probability, we run this procedure $t$ times and obtain all the outputs. Among the $t=2 \log (2 / \delta)$ runs of Algorithm 2, we have that $v_{\text {max }}$ is never compared with any value of $C$ in atleast one of the iterations with a probability at least

$$
1-(1-1 / 2)^{2 \log (2 / \delta)} \geq 1-\frac{\delta}{2}
$$

Hence, $T=\cup_{i} T_{i}$ contains $v_{\max }$ with a probability $1-\frac{\delta}{2}$.

Theorem 8.8 (Theorem 3.6 Restated). Given a set of values $V$, Algorithm 4 returns $a(1+\mu)^{3}$ approximation of maximum value with probability $1-\delta$ using $O\left(n \log ^{2}(1 / \delta)\right)$ oracle queries.

Proof. In Algorithm 4, we first identify an approximate maximum value using Sampling. If $|C| \geq \frac{\sqrt{n}}{2}$, then, from Lemma 8.5 , we have that the value returned is a $(1+\mu)$ approximation of the maximum value of $V$. Othwerwise, from Lemma 8.7, $T$ contains $v_{\text {max }}$ with a probability $1-\delta / 2$. As we use Count-MAx on the set $\widetilde{V} \cup T$, we know that the value returned, i.e., $u_{\max }$ is a $(1+\mu)^{2}$ of the maximum among values from $\widetilde{V} \cup T$. Therefore, $u_{\max } \geq \frac{v_{\max }}{(1+\mu)^{3}}$. Using union bound, the total probability of failure is $\delta$.

For query complexity, Algorithm 3 obtains a set $\widetilde{V}$ of $\sqrt{n} t$ sample values. Along with the set $T$ obtained (where $|T|=\frac{n t}{l}$ ), we use Count-Max on $\widetilde{V} \cup T$ to output the maximum $u_{\max }$. This step requires $O\left(|\widetilde{V} \cup T|^{2}\right)=O\left(\left(\sqrt{n} t+\frac{n t}{l}\right)^{2}\right)$ oracle queries. In an iteration $i$, for obtaining $T_{i}$, we make $O\left(\sum_{j}\left|V_{j}\right|\right)=O(n)$ oracle queries (Claim 8.2), and for $t$ iterations, we make $O(n t)$ queries. Using $t=2 \log (2 / \delta), l=\sqrt{n}$, in total, we make $O\left(n t+\left(\sqrt{n} t+\frac{n t}{l}\right)^{2}\right)=O\left(n \log ^{2}(1 / \delta)\right)$ oracle queries. Hence, the theorem.

\subsection{Probabilistic Noise}

LEMma 8.9. Suppose the maximum value $u_{\text {max }}$ is returned by Algorithm 2 with parameters $(V, n)$. Then, $\operatorname{rank}\left(u_{\max }, V\right)=O(\sqrt{n \log (1 / \delta)})$ with a probability of $1-\delta$.

Proof. We have for the maximum value $v_{\max }$, expected count value :

$$
\mathbf{E}\left[\operatorname{Count}\left(v_{\max }, V\right)\right]=\sum_{w \in V} 1\left\{O\left(w, v_{\max }\right)==w\right\}=(n-1)(1-p)
$$


Using Hoeffding's inequality, with probability $1-\delta / 2$ :

$$
\operatorname{Count}\left(v_{\max }, V\right) \geq(n-1)(1-p)-\sqrt{((n-1) \log (2 / \delta)) / 2}
$$

Consider a record $u \in V$ with rank at most $5 \sqrt{2 n \log (2 / \delta)}$. Then,

$$
\mathrm{E}[\operatorname{Count}(u, V)]=\sum_{w \in V} 1\left\{O\left(u, v_{\max }\right)==w\right\}=(n-\operatorname{rank}(u))(1-p)+(\operatorname{rank}(u)-1) p
$$

Using Hoeffding's inequality, with probability $1-\delta / 2$ :

$$
\begin{aligned}
\operatorname{Count}(u, V) & <(n-1)(1-p)-(\operatorname{rank}(u)-1)(1-2 p)+\sqrt{0.5(n-1) \log (2 / \delta)} \\
& <(n-1)(1-p)-(5 \sqrt{2 n \log (2 / \delta)}-1)(1-2 p)+\sqrt{0.5(n-1) \log (2 / \delta)} \\
& <\operatorname{Count}\left(v_{\max }, V\right)
\end{aligned}
$$

The last inequality is true for a value of $p \leq 0.4$. As Algorithm 2 returns the record $u_{\max }$ with maximum Count value, we have that $\operatorname{rank}\left(u_{\max }, V\right)=O(\sqrt{n \log (1 / \delta)})$. Using union bound, for the above conditions to be met, we have the claim.

To improve the query complexity, we use an early stopping criteria that discards a value $x$ using the Count $(x, V)$ when it determines that $x$ has no chance of being the maximum. Algorithm 12 presents the psuedocode for this modified count calculation. We sample $100 \log (n / \delta)$ values randomly, denoted by $S_{t}$ and compare every non-sampled point with $S_{t}$. We argue that by doing so, it helps us eliminate the values that are far away from the maximum in the sorted ranking. Using Algorithm 12, we compare the Count scores with respect to $S_{t}$ of a value $u \in V \backslash S_{t}$ and if Count $\left(u, S_{t}\right) \geq 50 \log (n / \delta)$, we make it available for the subsequent iterations.

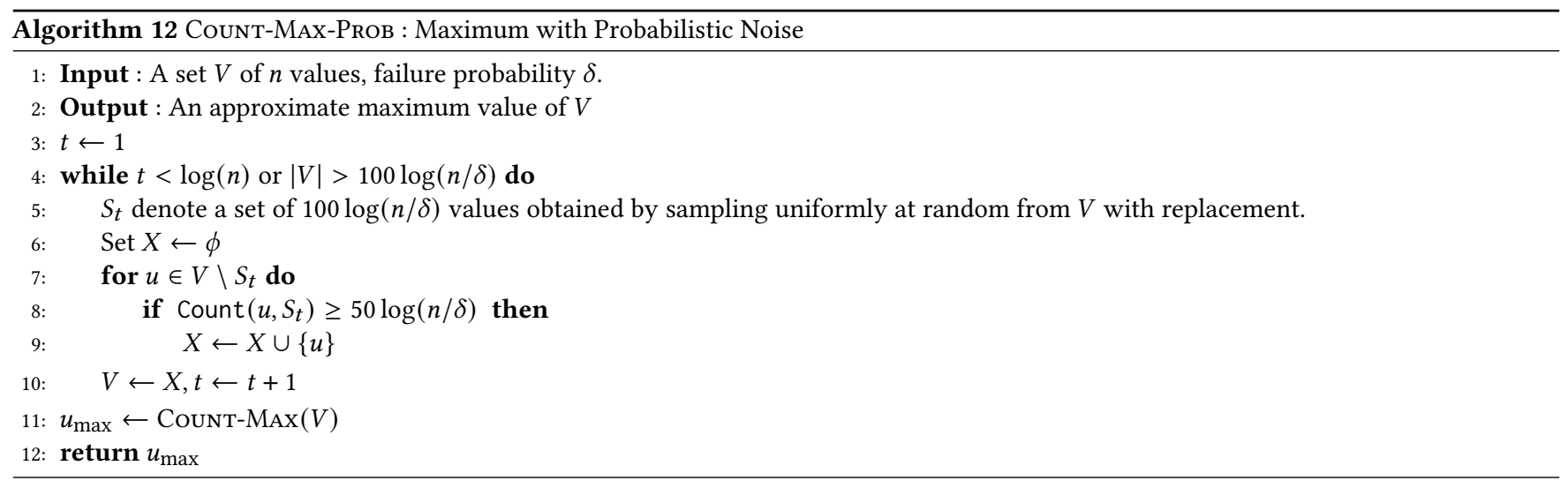

As Algorithm 12 considers each value $u \in V \backslash S_{t}$ by iteratively comparing it with each value $x \in S_{t}$ and the error probability is less than $p$, the expected count of $v_{\max }$ (if it is available) at any iteration $t$ is $(1-p)\left|S_{t}\right|$. Accounting for the deviation around the expected value, we have that the Count $\left(v_{\max }, S_{t}\right)$ is at least $50 \log (n / \delta)$ when $p \leq 0.4^{4}$. If a particular value $u$ has Count $\left(u, S_{t}\right)<50 \log (n / \delta)$ in any iteration, i.e., then it can not be the largest value in $V$ and therefore, we remove it from the set of possible candidates for maximum. Therefore, any value that remains in $V$ after an iteration $t$, must have rank closer to that of $v_{\max }$. We argue that after every iteration, the number of candidates remaining is at most $1 / 60$ th of the possible candidates.

LEMMA 8.10. In an iteration $t$ containing $n_{t}$ remaining records, using Algorithm 5 , with probability $1-\delta / n$, we discard at least $\frac{59}{60} \cdot n_{t}$ records.

Proof. Consider an iteration $t$ which has $n_{t}$ remaining records. Algorithm 5 and a record $u$ with rank $\alpha \cdot n_{t}$. Now, we have :

$$
\mathrm{E}\left[\operatorname{Count}\left(u, S_{t}\right)\right]=((1-\alpha)(1-p)+\alpha p) 100 \log (n / \delta)
$$

For $\alpha=0$, i.e., we have for maximum value $v_{\max }$

$$
\mathrm{E}\left[\operatorname{Count}\left(v_{\max }, S_{t}\right)\right]=(1-p) 100 \log (n / \delta)
$$

Using $p \leq 0.4$ and Hoeffding's inequality, with probability $1-\delta / n^{2}$, we have :

$$
\operatorname{Count}\left(v_{\max }, S_{t}\right) \geq(1-p) 100 \log (n / \delta)-\sqrt{100} \log (n / \delta) \geq 50 \log (n / \delta)
$$

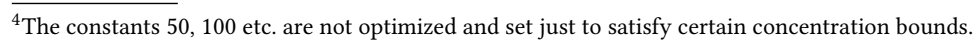


For $u$, we calculate the Count value. Using $p \leq 0.4$ and Hoeffding's inequality, with probability $1-\delta / n^{2}$, we have :

$$
\begin{aligned}
\operatorname{Count}\left(u, S_{t}\right) & <((1-\alpha)(1-p)+\alpha p) 100 \log (n / \delta)+\sqrt{100((1-\alpha)(1-p)+\alpha p)} \log (n / \delta) \\
& <((1-0.6 \alpha) 100+\sqrt{100(1-0.6 \alpha)}) \log (n / \delta)<50 \log (n / \delta)
\end{aligned}
$$

Upon calculation, for $\alpha>\frac{59}{60}$, we have the above expression. Therefore, using union bound, with probability $1-O(\delta / n)$, all records $u$ with rank at least $\frac{59 n_{t}}{60}$ satisfy :

$$
\operatorname{Count}\left(u, S_{t}\right)<\operatorname{Count}\left(v_{\max }, S_{t}\right)
$$

So, all such values can be removed. Hence, the claim.

In the previous lemma, we argued that in every iteration, at least $1 / 60$ th fraction is removed and therefore in $\Theta(\log n)$ iterations, the algorithm will terminate. In each iteration, we discard the sampled values $S_{t}$ to ensure that there is no dependency between the Count scores, and our guarantees hold. As we remove at most $O(t \cdot \log (n / \delta))=O\left(\log ^{2}(n / \delta)\right)$ sampled points, our final statement of the result is :

LemmA 8.11. Query complexity of Algorithm 5 is $O\left(n \cdot \log ^{2}(n / \delta)\right)$ and $u_{\max }$ satisfies rank $\left(u_{\max }, V\right) \leq O\left(\log ^{2}(n / \delta)\right)$ with probability $1-\delta$.

Proof. From Lemma 8.10, we have with probability $1-\delta / n$, after iteration $t$, at least $\frac{59 n_{t}}{60}$ records removed along with the $100 \log (n / \delta)$ records that are sampled. Therefore, we have :

$$
n_{t+1} \leq \frac{n_{t}}{60}-100 \log (n / \delta)
$$

After $\log (n / \delta)$ iterations, we have $n_{t+1} \leq 1$. As we have removed $\log _{60} n \cdot 100 \log (n / \delta)$ records that were sampled in total, these could records with rank $\leq 100 \log ^{2}(n / \delta)$. So, the rank of $u_{\max }$ output is at most $100 \log ^{2}(n / \delta)$. In an iteration $t$, the number of oracle queries calculating Count values is $O\left(n_{t} \cdot \log (n / \delta)\right)$. In total, Algorithm 5 makes $O\left(n \log ^{2}(n / \delta)\right)$ oracle queries. Using union bound over $\log (n / \delta)$ iterations, we get a total failure probability of $\delta$.

THEOREM 8.12 (TheOREM 3.7 RESTATED). There is an algorithm that returns $u_{\text {max }} \in V$ such that rank $\left(u_{\max }, V\right)=O\left(\log ^{2}(n / \delta)\right)$ with probability $1-\delta$ and requires $O\left(n \log ^{2}(n / \delta)\right)$ oracle queries.

Proof. The proof follows from Lemma 8.11

\section{FARTHEST AND NEAREST NEIGHBOR}

Lemma 9.1 (Lemma 3.9 Restated). Suppose $\max _{v_{i} \in S} d\left(u, v_{i}\right) \leq \alpha$ and $|S| \geq 6 \log (1 / \delta)$. Consider two records $v_{i}$ and $v_{j}$ such that $d\left(u, v_{i}\right)<$ $d\left(u, v_{j}\right)-2 \alpha$ then FCount $\left(v_{i}, v_{j}\right) \geq 0.3|S|$ with a probability of $1-\delta$

Proof. Since $d\left(u, v_{i}\right)<d\left(u, v_{j}\right)-2 \alpha$, for a point $x \in S$,

$$
\begin{aligned}
d\left(v_{j}, x\right) & \geq d\left(u, v_{j}\right)-d(u, x) \\
& >d\left(u, v_{i}\right)+2 \alpha-d(u, x) \\
& \geq d\left(v_{i}, x\right)-d(u, x)+2 \alpha-d(u, x) \\
& \geq d\left(v_{i}, x\right)+2 \alpha-2 d(u, x) \\
& \geq d\left(v_{i}, x\right)
\end{aligned}
$$

So, $O\left(v_{i}, x, v_{j}, x\right)$ is No with a probability $p$. As $p \leq 0.4$, we have :

$$
\begin{aligned}
\mathrm{E}\left[\mathrm{FCount}\left(v_{i}, v_{j}\right)\right] & =(1-p)|S| \\
\operatorname{Pr}\left[\mathrm{FCount}\left(v_{i}, v_{j}\right) \leq 0.3|S|\right] & \leq \operatorname{Pr}\left[\mathrm{FCount}\left(v_{i}, v_{j}\right) \leq(1-p)|S| / 2\right]
\end{aligned}
$$

From Hoeffding's inequality (with binary random variables), we have with a probability $\exp \left(-\frac{|S|(1-p)^{2}}{2}\right) \leq \delta(\operatorname{using}|S| \geq 6 \log (1 / \delta), p<0.4)$ : FCount $\left(v_{i}, v_{j}\right) \leq(1-p)|S| / 2$. Therefore, with probability at most $\delta$, we have, FCount $\left(v_{i}, v_{j}\right) \leq 0.3|S|$.

For the sake of completeness, we restate the Count definition that is used in Algorithm Count-Max. For every oracle comparison, we replace it with the pairwise comparison query described in Section 3.3. Let $u$ be a query point and $S$ denote a set of $\Theta(\log (n / \delta))$ points within a distance of $\alpha$ from $u$. We maintain a Count score for a given point $v_{i} \in V$ as :

$$
\operatorname{Count}\left(u, v_{i}, S, V\right)=\sum_{v_{j} \in V \backslash\left\{v_{i}\right\}} 1\left\{\operatorname{PAIRWISE-COMP}\left(u, v_{i}, v_{j}, S\right)==\text { No }\right\}
$$

Lemma 9.2. Given a query vertex $u$ and a set $S$ with $|S|=\Omega(\log (n / \delta))$ such that $\max _{v \in S} d(u, v) \leq \alpha$. Then the farthest identified using Algorithm 13 (with PAIRWISECOMP), denoted by $u_{\max }$ is within $4 \alpha$ distance from the optimal farthest point, i.e., $d\left(u, u_{\max }\right) \geq \max _{v \in V} d(u, v)-4 \alpha$ with a probability of $1-\delta$. Further the query complexity is $O\left(n^{2} \log (n / \delta)\right)$. 


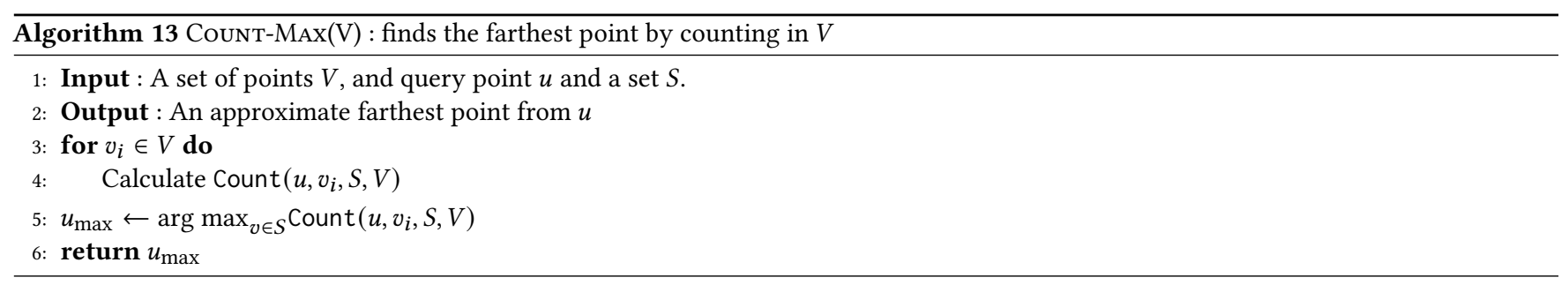

Proof. Let $v_{\max }=\max _{v \in V} d(u, v)$. Consider a value $w \in V$ such that $d(u, w)<d\left(u, v_{\max }\right)-4 \alpha$. We compare the Count values for $v_{\max }$ and $w$ given by, Count $\left(u, v_{\max }, S, V\right)=\sum_{v_{j} \in V \backslash\left\{v_{\max }\right\}} 1\left\{\operatorname{PAIRwiSE-Comp}\left(u, v_{\max }, v_{j}, S\right)==\operatorname{No}\right\}$ and $\operatorname{Count}(u, w, S, V)=$ $\sum_{v_{j} \in V \backslash\{w\}} 1\left\{\operatorname{PAIRwiSe-Comp}\left(u, w, v_{j}, S\right)==\right.$ No\}. We argue that $w$ can never be returned by Algorithm 13, i.e., Count $(u, w, S, V)<$ Count $\left(u, v_{\max }, S, V\right)$. Using Lemma 9.1 we have :

$$
\begin{aligned}
\operatorname{Count}\left(u, v_{\max }, S, V\right) & =\sum_{v_{j} \in V \backslash\left\{v_{\max }\right\}} 1\left\{\operatorname{PAIRwISE-COMP}\left(u, v_{\max }, v_{j}, S\right)==\text { No }\right\} \\
& \geq \sum_{v_{j} \in V \backslash\left\{v_{\max }\right\}} 1\left\{d\left(u, v_{j}\right)<d\left(u, v_{\max }\right)-2 \alpha\right\} \\
& =1\left\{d(u, w)<d\left(u, v_{\max }\right)-2 \alpha\right\}+\sum_{v_{j} \in V \backslash\left\{v_{\max }, w\right\}} 1\left\{d\left(u, v_{j}\right)<d\left(u, v_{\max }\right)-2 \alpha\right\} \\
& =1+\sum_{v_{j} \in V \backslash\left\{v_{\max }, w\right\}} 1\left\{d\left(u, v_{j}\right)<d\left(u, v_{\max }\right)-2 \alpha\right\} \\
\operatorname{Count}(u, w, S, V) & =\sum_{v_{j} \in V \backslash\{w\}} 1\left\{\operatorname{PAIRwISE}-\operatorname{Comp}\left(u, w, v_{j}, S\right)==\text { No }\right\} \\
& \leq \sum_{v_{j} \in V \backslash\{w\}} 1\left\{d\left(u, v_{j}\right)<d(u, w)+2 \alpha\right\} \\
& \leq \sum_{v_{j} \in V \backslash\left\{w, v_{\max }\right\}} 1\left\{d\left(u, v_{j}\right)<d\left(u, v_{\max }\right)-2 \alpha\right\}
\end{aligned}
$$

Combining the two, we have :

$$
\operatorname{Count}\left(u, v_{\max }, S, V\right)>\operatorname{Count}(u, w, S, V)
$$

This shows that the Countof $v_{\max }$ is strictly greater than the count of any point $w$ when $d(u, w)<d\left(u, v_{\max }\right)-4 \alpha$. Therefore, our algorithm would have output $v_{\max }$ instead of $w$. For calculating the Count for all points in $V$, we make at most $|V|^{2} \cdot|S|$ oracle queries as we compare every point with every other point using Algorithm 5. Finally, we output the point $u_{\max }$ as the value with highest Count. From Lemma 9.1, when $|S|=\Omega(\log (n / \delta))$, the answer to any pairwise query is correct with a failure probability of $\delta / n^{2}$. As there are $n^{2}$ pairwise comparisons, and each with failure probability of $\delta / n^{2}$, from union bound, we have that that the total failure probability is $\delta$. Hence, the claim.

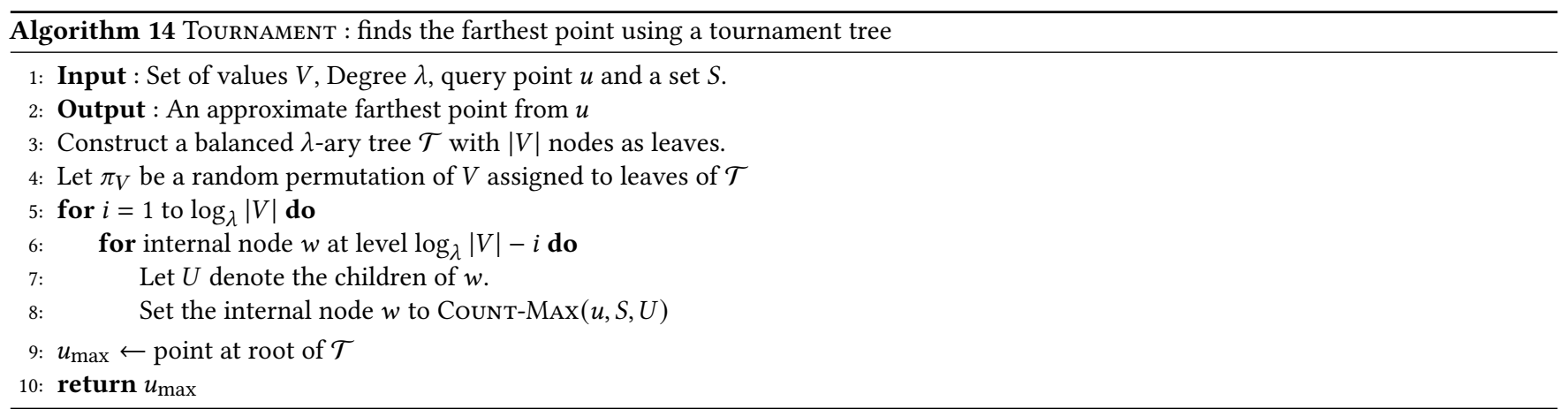

Let the farthest point from query point $u$ among $V$ be denoted by $v_{\max }$ and the set of records for which the oracle answer can be incorrect is given by

$$
C=\left\{w \mid w \in V, d(u, w) \geq d\left(u, v_{\max }\right)-2 \alpha\right\}
$$



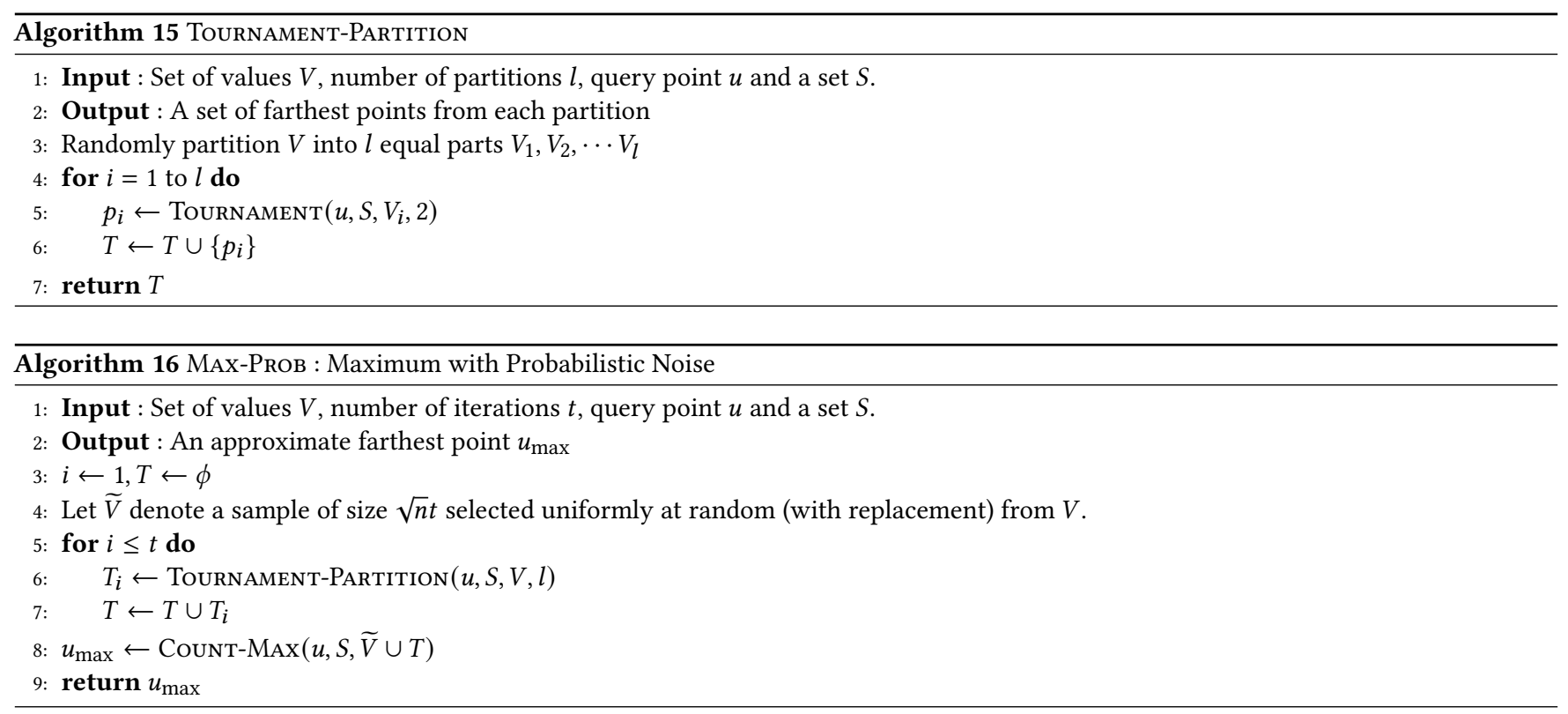

LEMma 9.3. (1) If $\rfloor C \mid>\sqrt{n} / 2$, then there exists a value $v_{j} \in \widetilde{V}$ satisfying $d\left(u, v_{j}\right) \geq d\left(u, v_{\text {max }}\right)-2 \alpha$ with a probability of $1-\delta / 2$.

(2) Suppose $|C| \leq \sqrt{n} / 2$. Then, $T$ contains $v_{\max }$ with a probability at least $1-\delta / 2$.

Proof. The proof is similar to Lemma 8.7.

THEOREM 9.4 (THEOREM 3.10 RESTATED). Given a query vertex $u$ and a set $S$ with $|S|=\Omega(\log (n / \delta))$ such that $\max _{v \in S} d(u, v) \leq \alpha$ then the farthest identified using Algorithm 4 (with PAIRWISECOMP), denoted by $u_{m a x}$ is within $6 \alpha$ distance from the optimal farthest point, i.e., $d\left(u, u_{\max }\right) \geq \max _{v \in V} d(u, v)-6 \alpha$ with a probability of $1-\delta$. Further the query complexity is $O\left(n \log ^{3}(n / \delta)\right)$.

Proof. The proof is similar to Theorem 8.8. In Algorithm 16, we first identify an approximate maximum value using Sampling. If $|C| \geq \frac{\sqrt{n}}{2}$, then, from Lemma 9.3, we have that the value returned is a $2 \alpha$ additive approximation of the maximum value of $V$. Otherwise, from Lemma 9.3, $T$ contains $v_{\max }$ with a probability $1-\delta / 2$. As we use CounT-MAx on the set $\widetilde{V} \cup T$, we know that the value returned, i.e., $u_{\max }$ is a $4 \alpha$ of the maximum among values from $\widetilde{V} \cup T$. Therefore, $d\left(u, u_{\max }\right) \geq d\left(u, v_{\max }\right)-6 \alpha$. Using union bound over $n \cdot t$ comparisons, the total probability of failure is $\delta$.

For query complexity, Algorithm 15 obtains a set $\widetilde{V}$ of $\sqrt{n} t$ sample values. Along with the set $T$ obtained (where $|T|=\frac{n t}{l}$ ), we use Count-Max on $\widetilde{V} \cup T$ to output the maximum $u_{\max }$. This step requires $O\left(|\widetilde{V} \cup T|^{2}|S|\right)=O\left(\left(\sqrt{n} t+\frac{n t}{l}\right)^{2} \log (n / \delta)\right)$ oracle queries. In an iteration $i$, for obtaining $T_{i}$, we make $O\left(\sum_{j}\left|V_{j}\right| \log (n / \delta)\right)=O(n \log (n / \delta))$ oracle queries (Claim 8.2), and for $t$ iterations, we make $O(n t \log (n / \delta))$ queries. Using $t=2 \log (2 n / \delta), l=\sqrt{n}$, in total, we make $O\left(n t \log (n / \delta)+\left(\sqrt{n} t+\frac{n t}{l}\right)^{2} \log (n / \delta)\right)=O\left(n \log { }^{3}(n / \delta)\right)$ oracle queries. Hence, the theorem.

\section{$10 k$-CENTER : ADVERSARIAL NOISE}

LEMMA 10.1. Suppose in an iteration $t$ of Greedy algorithm, centers are given by $S_{t}$ and we reassign points using AssigN which is a $\beta$-approximation to the correct assignment. In iteration $t+1$, using this assignment, if we obtain an $\alpha$-approximate farthest point using APPROX-FARTHEST, then, after $k$ iterations, Greedy algorithm obtains a $2 \alpha \beta^{2}$-approximation for the $k$-center objective.

Proof. Consider an optimum clustering $C^{*}$ with centers $u_{1}, u_{2}, . ., u_{k}$ respectively: $C^{*}\left(u_{1}\right), C^{*}\left(u_{2}\right), \cdots, C^{*}\left(u_{k}\right)$. Let the centers obtained by Algorithm 6 be denoted by $S$. If $\left|S \cap C^{*}\left(u_{i}\right)\right|=1$ for all $i$, then, for some point $x \in C^{*}\left(u_{i}\right)$ assigned to $s_{j} \in S$ by Algorithm Assign, we have

$$
\begin{aligned}
& d\left(x, S \cap C^{*}\left(u_{i}\right)\right) \leq d\left(x, u_{i}\right)+d\left(u_{i}, S \cap C^{*}\left(u_{i}\right)\right) \leq 2 O P T \\
& \Longrightarrow d\left(x, s_{j}\right) \leq \beta \min _{s_{k} \in S} d\left(x, s_{k}\right) \leq \beta d\left(x, S \cap C^{*}\left(u_{i}\right)\right) \leq 2 \beta O P T
\end{aligned}
$$

Therefore, every point in $V$ is at a distance of at most $2 \beta O P T$ from a center assigned in $S$.

Suppose for some $j$ we have $\left|S \cap C^{*}\left(u_{j}\right)\right| \geq 2$. Let $s_{1}, s_{2} \in S \cap C^{*}\left(u_{j}\right)$ and $s_{2}$ appeared after $s_{1}$ in iteration $t+1$. As $s_{1} \in S_{t}$, we have $\min _{w \in S_{t}} d\left(w, s_{2}\right) \leq d\left(s_{1}, s_{2}\right)$. In iteration $t$, we know that the farthest point $s_{2}$ is an $\alpha$-approximation of the farthest point (say $f_{t}$ ). Moreover, suppose $s_{2}$ assigned to cluster with center $s_{k}$ in iteration $t$ that is a $\beta$-approximation of it's true center. Therefore,

$$
\frac{1}{\alpha} \min _{w \in S_{t}} d\left(w, f_{t}\right) \leq d\left(s_{k}, s_{2}\right) \leq \beta \min _{w \in S_{t}} d\left(w, s_{2}\right) \leq \beta d\left(s_{1}, s_{2}\right)
$$


Because $s_{1}$ and $s_{2}$ are in the same optimum cluster, from triangle inequality we have $d\left(s_{1}, s_{2}\right) \leq 2 O P T$. Combining all the above we get $\min _{w \in S_{t}} d\left(w, f_{t}\right) \leq 2 \alpha \beta O P T$ which means that farthest point of iteration $t$ is at a distance of $2 \alpha \beta O P T$ from $S_{t}$. In the subsequent iterations, the distance of any point to the final set of centers, given by $S$ only gets smaller. Hence,

$$
\max _{v} \min _{w \in S} d(v, w) \leq \max _{v} \min _{w \in S_{t}} d(v, w)=\min _{w \in S_{t}} d\left(f_{t}, w\right) \leq 2 \alpha \beta O P T
$$

However, when we output the final clusters and centers, the farthest point after $k$-iterations (say $f_{k}$ ) could be assigned to center $v_{j} \in S$ that is a $\beta$-approximation of the distance to true center.

$$
d\left(f_{k}, v_{j}\right) \leq \beta \min _{w \in S} d\left(f_{k}, w\right) \leq 2 \alpha \beta^{2} \text { OPT }
$$

Therefore, every point is assigned to a cluster with distance at most $2 \alpha \beta^{2}$ OPT. Hence the claim.

LEMma 10.2. Given a set $S$ of centers, Algorithm AssigN assigns a point $u$ to a cluster $s_{j} \in S$ such that $d\left(u, s_{j}\right) \leq(1+\mu)^{2} \min _{s_{t} \in S}\left\{d\left(u, s_{t}\right)\right\}$ using $O(n k)$ queries.

Proof. The proof is essentially the same as Lemma 8.3 and uses MCount instead of Count.

LEMma 10.3. Given a set of centers $S$, Algorithm 4 identifies a point $v_{j}$ with probability $1-\delta / k$, such that

$$
\min _{s_{j} \in S} d\left(v_{j}, s_{j}\right) \geq \max _{v_{t} \in V} \min _{s_{t} \in S} \frac{d\left(v_{t}, s_{t}\right)}{(1+\mu)^{5}}
$$

Proof. Suppose $v_{t}$ is the farthest point assigned to center $s_{t} \in S$. Let $v_{j}$, assigned to $s_{j} \in S$ be the point returned by Algorithm 4 . From Theorem 8.8, we have :

$$
\begin{aligned}
d\left(v_{j}, s_{j}\right) & \geq \frac{\max _{v_{i} \in V} d\left(v_{i}, s_{i}\right)}{(1+\mu)^{3}} \\
& \geq \frac{d\left(v_{t}, s_{t}\right)}{(1+\mu)^{3}} \geq \frac{\min _{s_{t}^{\prime} \in S} d\left(v_{t}, s_{t}^{\prime}\right)}{(1+\mu)^{3}}
\end{aligned}
$$

Due to error in assignment, using Lemma 10.2

$$
d\left(v_{j}, s_{j}\right) \leq(1+\mu)^{2} \min _{s_{j}^{\prime} \in S} d\left(v_{j}, s_{j}^{\prime}\right)
$$

Combining the above equations we have

$$
\min _{s_{j}^{\prime} \in S} d\left(v_{j}, s_{j}^{\prime}\right) \geq \frac{\min _{s_{t}^{\prime} \in S} d\left(v_{t}, s_{t}^{\prime}\right)}{(1+\mu)^{5}}
$$

For Approx-Farthest, we use $l=\sqrt{n}$ and $t=\log (2 k / \delta)$ and $\widetilde{V}=\sqrt{n} t$. So, following the proof in Theorem 3.6, we succeed with probability $1-\delta / k$. Hence, the lemma.

LEMMA 10.4. Given a current set of centers $S$,

(1) Assign assigns a point $u$ to a cluster $C\left(s_{i}\right)$ such that $d\left(u, s_{i}\right) \leq(1+\mu)^{2} \min _{s_{j} \in S}\left\{d\left(u, s_{j}\right)\right\}$ using $O(n k)$ oracle queries additionally.

(2) APPRoX-FARTHEST identifies a point $w$ in cluster $C\left(s_{i}\right)$ such that $\min _{s_{j} \in S} d\left(w, s_{j}\right) \geq \max _{v_{t} \in V} \min _{s_{t} \in S} d\left(v_{t}, s_{t}\right) /(1+\mu)^{5}$ with probability $1-\frac{\delta}{k}$ using $O\left(n \log ^{2}(k / \delta)\right)$ oracle queries.

Proof. (1) From Lemma 10.2, we have the claim. We assign a point to a cluster based on the scores the cluster center received in comparison to other centers. Except for the newly created center, we have previously queried every center with every other center. Therefore, number of new oracle queries made for every point is $O(k)$; that gives us a total of $O(n k)$ additional new queries used by AssigN.

(2) From Lemma 10.3, we have that $\min _{s_{j} \in S} d\left(w, s_{j}\right) \geq \max _{v_{t} \in V} \min _{s_{t} \in S} \frac{d\left(v_{t}, s_{t}\right)}{(1+\mu)^{5}}$ with probability $1-\delta / k$. As the total number of queries made by Algorithm 4 is $O\left(n t+\left(\frac{n t}{l}+\sqrt{n} t\right)^{2}\right)$. For Approx-FArthest, we use $l=\sqrt{n}$ and $t=\log (2 k / \delta)$ and $\widetilde{V}=\sqrt{n} t$, therefore, the query complexity is $O\left(n \log ^{2}(k / \delta)\right)$.

Theorem 10.5 (Theorem 4.2 Restated). For $\mu<\frac{1}{18}$, Algorithm 6 achieves a $(2+O(\mu))$-approximation for the $k$-center objective using $O\left(n k^{2}+n k \cdot \log ^{2}(k / \delta)\right)$ oracle queries with probability $1-\delta$.

Proof. From the above discussed claim and Lemma 10.4, we have that Algorithm 6 achieves a $2(1+\mu)^{9}$ approximation for $k$-center objective. When $\mu<\frac{1}{18}$, we can simplify the approximation factor to $2+18 \mu$, i.e., $2+O(\mu)$. From Lemma 10.4 , we have that in each iteration, we succeed with probability $1-\delta / k$. Using union bound, the failure probability is given by $\delta$. For query complexity, as there are $k$ iterations, and in each iteration we use Assign and Approx-FArthest, using Lemma 10.4, we have the theorem. 


\section{$11 k$-CENTER : PROBABILISTIC NOISE}

\subsection{Sampling}

Lemma 11.1. Consider the sample $\widetilde{V} \subseteq V$ of points obtained by selecting each point with a probability $\frac{450 \log (n / \delta)}{m}$. Then, we have $\frac{400 n \log (n / \delta)}{m} \leq$ $|\widetilde{V}| \leq \frac{500 n \log (n / \delta)}{m}$ and for every $i \in[k],\left|C^{*}\left(s_{i}\right) \cap \widetilde{V}\right| \geq 400 \log (n / \delta)$ with probability $1-O(\delta)$ for sufficiently large $\gamma>0$.

Proof. We include every point in $\widetilde{V}$ with a probability $\frac{450 \log (n / \delta)}{m}$ where the size of the smallest cluster is $m$. Using Chernoff bound, with probability $1-O(\delta)$, we have :

$$
\frac{400 n \log (n / \delta)}{m} \leq|\widetilde{V}| \leq \frac{500 n \log (n / \delta)}{m}
$$

Consider an optimal cluster $C^{*}\left(v_{i}\right)$ with center $v_{i}$. As every point is included with probability $\frac{450 \log (n / \delta)}{m}$ :

$$
\mathrm{E}\left[\left|C^{*}\left(s_{i}\right) \cap \widetilde{V}\right|\right]=\left|C^{*}\left(s_{i}\right)\right| \cdot \frac{450 \log (n / \delta)}{m} \geq 450 \log (n / \delta)
$$

Using Chernoff bound, with probability at least $1-\delta / n$, we have

$$
\left|C^{*}\left(s_{i}\right) \cap \tilde{V}\right| \geq 400 \log (n / \delta)
$$

Using union bound for all the $k$ clusters, we have the lemma.

\subsection{Assignment}

$$
\operatorname{ACount}\left(u, s_{i}, s_{j}\right)=\sum_{x \in R\left(s_{i}\right)} 1\left\{O\left(u, x, u, s_{j}\right)==\text { Yes }\right\}
$$

LEMmA 11.2. Consider a point $u$ and $s_{j} \neq s_{i}$ such that $d\left(u, s_{i}\right) \leq d\left(u, s_{j}\right)-2$ OPT and $\left|R\left(s_{i}\right)\right| \geq 12 \log (n / \delta)$, then, ACount $\left(u, s_{i}, s_{j}\right) \geq 0.3\left|R\left(s_{i}\right)\right|$ with a probability of $1-\frac{\delta}{n^{2}}$.

Proof. Using triangle inequality, for any $x \in R\left(s_{i}\right)$

$$
d(u, x) \leq d\left(u, s_{i}\right)+d\left(s_{i}, x\right) \leq d\left(u, s_{j}\right)-2 \mathrm{OPT}+d\left(s_{i}, x\right) \leq d\left(u, s_{j}\right)
$$

So, $O\left(u, x, u, s_{j}\right)$ is Yes with a probability at least $1-p$. We have:

$$
\mathrm{E}\left[\operatorname{ACount}\left(u, s_{i}, s_{j}\right)\right]=\sum_{x \in R\left(s_{i}\right)} \mathrm{E}\left[1\left\{O\left(u, x, u, s_{j}\right)==Y \mathrm{Yes}\right\}\right] \geq(1-p)\left|R\left(s_{i}\right)\right|
$$

Using Hoeffding's inequality, with a probability of $\exp \left(-\left|R\left(s_{i}\right)\right|(1-p)^{2} / 2\right) \leq \frac{\delta}{n^{2}}$ (using $p \leq 0.4$ ), we have

$$
\operatorname{ACount}\left(u, s_{i}, s_{j}\right) \leq(1-p)\left|R\left(s_{i}\right)\right| / 2
$$

We have $\operatorname{Pr}\left[\operatorname{ACount}\left(u, s_{i}, s_{j}\right) \leq 0.3|S|\right] \leq \operatorname{Pr}\left[\operatorname{ACount}\left(u, s_{i}, s_{j}\right) \leq(1-p)|S| / 2\right]$. Therefore, with probability $\frac{\delta}{n^{2}}$, we have ACount $\left(u, s_{i}, s_{j}\right) \leq$ $0.3|S|$. Hence, the lemma.

Lemma 11.3. Suppose $u \in C^{*}\left(s_{i}\right)$ and for some $s_{j} \in S$, if $d\left(s_{i}, s_{j}\right) \geq 6 \mathrm{OPT}$, then, Algorithm 8 assigns $u$ to center $s_{i}$ with probability $1-\frac{\delta}{n^{2}}$.

Proof. As $u \in C^{*}\left(s_{i}\right)$, we have $d\left(u, s_{i}\right) \leq 2$ OPT. Therefore,

$$
\begin{aligned}
& d\left(s_{j}, u\right)-d\left(s_{i}, u\right) \geq d\left(s_{i}, s_{j}\right)-2 d\left(s_{i}, u\right) \geq 2 \mathrm{OPT} \\
& d\left(s_{j}, u\right) \geq d\left(s_{i}, u\right)+2 \mathrm{OPT}
\end{aligned}
$$

From Lemma 11.2, we have that if $d\left(u, s_{i}\right) \leq d\left(u, s_{j}\right)-2$ OPT, then, we will assign $u$ to $s_{i}$ with probability $1-\frac{\delta}{n^{2}}$.

LEMma 11.4. Given a set of centers $S$, every $u \in V$ is assigned to a cluster $s_{i}$ such that $d\left(u, s_{i}\right) \leq \min _{s_{j} \in S} d\left(u, s_{j}\right)+2 \mathrm{OPT}$ with a probability of $1-1 / n^{2}$.

Proof. From Lemma 11.2, we have that a point $u$ is assigned to $s_{l}$ from $s_{m}$ if $d\left(u, s_{l}\right) \leq d\left(u, s_{m}\right)-2$ OPT. If $s_{i}$ is the final assigned center of $u$, then, for every $s_{j}$, it must be true that $d\left(u, s_{j}\right) \geq d\left(u, s_{i}\right)-2$ OPT, which implies $d\left(u, s_{i}\right) \leq \min _{s_{j} \in S} d\left(u, s_{j}\right)+2$ OPT. Using union bound over at most $n$ points, we have with a probability of $1-\frac{\delta}{n}$, every point $u$ is assigned as claimed. 


\subsection{Core Calculation}

Consider a cluster $C\left(s_{i}\right)$ with center $s_{i}$. Let $S_{a}^{b}$ denote the number of points in the set $\left|\left\{x: a \leq d\left(x, s_{i}\right)<b\right\}\right|$.

$$
\operatorname{Count}(u)=\sum_{x \in C\left(s_{i}\right)} 1\left\{O\left(s_{i}, x, s_{i}, u\right)==\text { No }\right\}
$$

LEMMA 11.5. Consider any two points $u_{1}, u_{2} \in C\left(s_{i}\right)$ such that $d\left(u_{1}, s_{i}\right) \leq d\left(u_{2}, s_{i}\right)$, then $\mathbf{E}\left[\operatorname{Count}\left(u_{1}\right)\right]-\mathbf{E}\left[\operatorname{Count}\left(u_{2}\right)\right]=(1-2 p) S_{d\left(u_{1}, s_{i}\right)}^{d\left(u_{i}\right)}$

Proof. For a point $u \in C\left(s_{i}\right)$

$$
\begin{gathered}
\mathrm{E}[\operatorname{Count}(u)]=\mathrm{E}\left[\sum_{x \in C\left(s_{i}\right)} 1\left\{O\left(s_{i}, x, s_{i}, u\right)==\mathrm{No}\right\}\right] \\
=S_{0}^{d\left(u, s_{i}\right)} p+S_{d\left(u, s_{i}\right)}^{\infty}(1-p) \\
\mathrm{E}\left[\operatorname{Count}\left(u_{1}\right)\right]-\mathrm{E}\left[\operatorname{Count}\left(u_{2}\right)\right]=\left(S_{0}^{d\left(u_{1}, s_{i}\right)} p+S_{d\left(u_{1}, s_{i}\right)}^{d\left(u_{2}, s_{i}\right)}(1-p)+S_{d\left(u_{2}, s_{i}\right)}^{\infty}(1-p)\right)-\left(S_{0}^{d\left(u_{1}, s_{i}\right)} p++S_{d\left(u_{1}, s_{i}\right)}^{d\left(u_{2}, s_{i}\right)} p+S_{d\left(u_{2}, s_{i}\right)}^{\infty}(1-p)\right) \\
=(1-2 p) S_{d\left(u_{1}, s_{i}\right)}^{d\left(u_{2}\right)}
\end{gathered}
$$

LEMmA 11.6. Consider any two points $u_{1}, u_{2} \in C\left(s_{i}\right)$ such that $d\left(u_{1}, s_{i}\right) \leq d\left(u_{2}, s_{i}\right)$ and $\left|S_{d\left(u_{1}, s_{i}\right)}^{d\left(u_{2}\right)}\right| \geq \sqrt{100\left|C\left(s_{i}\right)\right| \log (n / \delta)}$. Then, Count $\left(u_{1}\right)>$ Count $\left(u_{2}\right)$ with probability $1-\delta / n^{2}$.

Proof. Suppose $u_{1}, u_{2} \in C\left(s_{i}\right)$. We have that Count $\left(u_{1}\right)$ and Count $\left(u_{2}\right)$ is a sum of $\left|C\left(s_{i}\right)\right|$ binary random variables.

Using Hoeffding's inequality, we have with probability $\exp \left(-\beta^{2} / 2\left|C\left(s_{i}\right)\right|\right)$ that

$$
\begin{aligned}
& \operatorname{Count}\left(u_{1}\right) \leq \mathrm{E}\left[\operatorname{Count}\left(u_{1}\right)\right]-\frac{\beta}{2} \\
& \operatorname{Count}\left(u_{2}\right)>\mathrm{E}\left[\operatorname{Count}\left(u_{2}\right)\right]+\frac{\beta}{2}
\end{aligned}
$$

Using union bound, with probability at least $1-2 \exp \left(-\beta^{2} / 2\left|C\left(s_{i}\right)\right|\right)$, we can conclude that

$$
\operatorname{Count}\left(u_{1}\right)-\operatorname{Count}\left(u_{2}\right)>\mathbf{E}\left[\operatorname{Count}\left(u_{1}\right)-\operatorname{Count}\left(u_{2}\right)\right]-\beta>(1-2 p) S_{d\left(u_{1}, s_{i}\right)}^{d\left(u_{2}, s_{i}\right)}-\beta
$$

Choosing $\beta=(1-2 p) S_{d\left(u_{1}, s_{i}\right)}^{d\left(u_{2}, s_{i}\right)}$, we have Count $\left(u_{1}\right)>\operatorname{Count}\left(u_{2}\right)$ with a probability (for constant $\left.p \leq 0.4\right)$

$$
1-2 \exp \left(-(1-2 p)^{2}\left(S_{d\left(u_{1}, s_{i}\right)}^{d\left(u_{2}, s_{i}\right)}\right)^{2} / 2\left|C\left(s_{i}\right)\right|\right) \geq 1-2 \exp \left(-0.02\left(S_{d\left(u_{1}, s_{i}\right)}^{d\left(u_{2}, s_{i}\right)}\right)^{2} /\left|C\left(s_{i}\right)\right|\right) .
$$

Further, simplifying using $S_{d\left(u_{1}, s_{i}\right)}^{d\left(u_{2}, s_{i}\right)} \geq \sqrt{100\left|C\left(s_{i}\right)\right| \log (n / \delta)}$, we get probability of failure is $2 \exp (-2 \log (n / \delta))=O\left(\delta / n^{2}\right)$

LEMmA 11.7. If $\left|C\left(s_{i}\right)\right| \geq 400 \log (n / \delta)$, then, $\left|R\left(s_{i}\right)\right| \geq 200 \log (n / \delta)$ with probability $1-\left|C\left(s_{i}\right)\right|^{2} \delta / n^{2}$.

Proof. From Lemma 11.6, we have that if there are points $u_{1}, u_{2}$ with $\sqrt{100\left|C\left(s_{i}\right)\right| \log (n / \delta)}$ many points between them, then, we can identify the closer one correctly. When $\left|C\left(s_{i}\right)\right| \geq 400 \log (n / \delta)$, we have $\sqrt{100\left|C\left(s_{i}\right)\right| \log (n / \delta)} \geq 200 \log (n / \delta)$ points between every point and the point with the rank $200 \log (n / \delta)$. Therefore, $\left|R\left(s_{i}\right)\right| \geq 200 \log (n / \delta)$. Using union bound over all pairs of points in the cluster, we get the claim.

LEMMA 11.8. If $x \in C^{*}\left(s_{i}\right)$, then, $x \in C\left(s_{i}\right)$ or $x$ is assigned to a cluster $s_{j}$ such that $d\left(x, s_{j}\right) \leq 8$ OPT.

Proof. If $x \in C^{*}\left(s_{i}\right)$, we argue that it will be assigned to $C\left(s_{i}\right)$. For the sake of contradiction, suppose $x$ is assigned to a cluster $C\left(s_{j}\right)$ for some $s_{j} \in S$. We have $d\left(x, s_{i}\right) \leq 2 \mathrm{OPT}$ and let $d\left(s_{i}, s_{j}\right) \geq 6 \mathrm{OPT}$

$$
\begin{gathered}
d\left(s_{i}, s_{j}\right) \leq d\left(s_{j}, x\right)+d\left(s_{i}, x\right) \\
d\left(s_{j}, x\right) \geq 4 \mathrm{OPT}
\end{gathered}
$$

However, we know that $d\left(s_{j}, x\right) \leq d\left(s_{i}, x\right)+2 \mathrm{OPT} \leq 4$ OPT from Lemma 11.2. We have a contradiction. Therefore, $x$ is assigned to $s_{i}$. If $d\left(s_{i}, s_{j}\right) \leq 6 \mathrm{OPT}$, we have $d\left(x, s_{j}\right) \leq d\left(x, s_{i}\right)+2 \mathrm{OPT} \leq 8 \mathrm{OPT}$. Hence, the lemma. 


\subsection{Farthest point computation}

Let $R\left(s_{i}\right)$ represent the core of the cluster $C\left(s_{i}\right)$ and contains $\Theta(\log (n / \delta))$ points. We define FCount for comparing two points $v_{i}, v_{j}$ from their centers $s_{i}, s_{j}$ respectively. If $s_{i} \neq s_{j}$, we let :

$$
\operatorname{FCount}\left(v_{i}, v_{j}\right)=\sum_{x \in \widetilde{R}\left(s_{i}\right), y \in \widetilde{R}\left(s_{j}\right)} 1\left\{O\left(v_{i}, x, v_{j}, y\right)==\text { Yes }\right\}
$$

Otherwise, we let FCount $\left(v_{i}, v_{j}\right)=\sum_{x \in R\left(s_{i}\right)} 1\left\{O\left(v_{i}, x, v_{j}, x\right)==\right.$ Yes $\}$. First, we observe that each of the summation is over $\left|R\left(s_{i}\right)\right|$ many terms, because $\left|\widetilde{R}\left(s_{i}\right)\right|=\sqrt{\left|R\left(s_{i}\right)\right|}$.

Lemma 11.9. Consider two records $v_{i}$, $v_{j}$ in different clusters $C\left(s_{i}\right), C\left(s_{j}\right)$ respectively such that $d\left(s_{i}, v_{i}\right)<d\left(s_{j}, v_{j}\right)-4 \mathrm{OPT}$ then FCount $\left(v_{i}, v_{j}\right) \geq 0.3\left|\widetilde{R}\left(s_{i}\right)\right|\left|\widetilde{R}\left(s_{j}\right)\right|$ with a probability of $1-\frac{\delta}{n^{2}}$.

Proof. We know $\max _{v_{i} \in \widetilde{R}\left(s_{i}\right)} d\left(u, v_{i}\right) \leq 2 \mathrm{OPT}$ and $\max _{v_{j} \in \widetilde{R}\left(s_{j}\right)} d\left(v_{j}, s_{j}\right) \leq 2 \mathrm{OPT}$.

For a point $x \in R\left(s_{i}\right), y \in R\left(s_{j}\right)$

$$
\begin{aligned}
d\left(v_{j}, y\right) & \geq d\left(s_{j}, v_{j}\right)-d\left(s_{j}, y\right) \\
& >d\left(v_{i}, s_{i}\right)+4 \mathrm{OPT}-d\left(s_{j}, y\right) \\
& >d\left(v_{i}, x\right)-d\left(x, s_{i}\right)+4 \mathrm{OPT}-d\left(s_{j}, y\right) \\
& >d\left(v_{i}, x\right)
\end{aligned}
$$

So, $O\left(v_{i}, x, v_{j}, y\right)$ is No with a probability $p$. As $p \leq 0.4$, we have :

$$
\begin{aligned}
\mathrm{E}\left[\mathrm{FCount}\left(v_{i}, v_{j}\right)\right] & =(1-p)\left|\widetilde{R}\left(s_{i}\right) \| \widetilde{R}\left(s_{j}\right)\right| \\
\operatorname{Pr}\left[\mathrm{FCount}\left(v_{i}, v_{j}\right) \leq 0.3\left|\widetilde{R}\left(s_{i}\right)\right|\left|\widetilde{R}\left(s_{j}\right)\right|\right] & \leq \operatorname{Pr}\left[\mathrm{FCount}\left(v_{i}, v_{j}\right) \leq(1-p)\left|\widetilde{R}\left(s_{i}\right)\right|\left|\widetilde{R}\left(s_{j}\right)\right| / 2\right]
\end{aligned}
$$

From Hoeffding's inequality (with binary random variables), we have with a probability $\exp \left(-\frac{\left|\widetilde{R}\left(s_{i}\right)\right|\left|\widetilde{R}\left(s_{j}\right)\right|(1-p)^{2}}{2}\right) \leq \frac{\delta}{n^{2}}($ using $\left.\left|\widetilde{R}\left(s_{i}\right) \| \widetilde{R}\left(s_{j}\right)\right| \geq 12 \log (n / \delta), p<0.4\right): \mathrm{FCount}\left(v_{i}, v_{j}\right) \leq(1-p)\left|\widetilde{R}\left(s_{i}\right) \| \widetilde{R}\left(s_{j}\right)\right| / 2$. Therefore, with probability at most $\delta / n^{2}$, we have, FCount $\left(v_{i}, v_{j}\right) \leq 0.3\left|\widetilde{R}\left(s_{i}\right)\right|\left|\widetilde{R}\left(s_{j}\right)\right|$.

In order to calculate the farthest point, we use the ideas discussed in Section 3 to identify the point that has the maximum distance from its assigned center. As noted in Section 3.3, our approximation guarantees dependend on the maximum distance of points in the core from the center. In the next lemma, we show that assuming a maximum distance of a point in the core (See Lemma 11.8), we can obtain a good approximation for the farthest point.

LEMMA 11.10. Let $\max _{s_{j} \in S, u \in R\left(s_{j}\right)} d\left(u, s_{j}\right) \leq \alpha$. In every iteration, if the farthest point is at a distance more than $(6 \alpha+3 \mathrm{OPT})$, then, APPROX-FARTHEST outputs a $(6 \alpha / \mathrm{OPT}+3)$-approximation. Otherwise, the point output is at most $(6 \alpha+3 \mathrm{OPT})$ away.

Proof. The farthest point output Approx-Farthest is a $6 \alpha$ additive approximation. However, the assignment of points to the cluster also introduces another additive approximation of 2 OPT, resulting in a total $6 \alpha+2$ OPT approximation. Suppose in the current iteration, the distance of the farthest point is $\beta$ OPT, then the point output by Approx-FArThest is at least $\beta$ OPT $-(6 \alpha+2$ OPT $)$ away. So, the approximation ratio is $\frac{\beta}{\beta-(6 \alpha+2 \mathrm{OPT})}$. If $\beta$ OPT $\geq 6 \alpha+3 \mathrm{OPT}$, we have $\frac{\beta \mathrm{OPT}}{\beta \mathrm{OPT}-(6 \alpha+2 \mathrm{OPT})} \leq \beta$. As we are trying to minimize the approximation ratio, we set $\beta \mathrm{OPT}=6 \alpha+3 \mathrm{OPT}$ and get the claimed guarantee.

\subsection{Final Guarantees}

Throughout this section, we assume that $m=\Omega\left(\frac{\log ^{3}(n / \delta)}{\delta}\right)$ for a given failure probability $\delta>0$.

LEMMA 11.11. Given a current set of centers $S$, and $\max _{v_{j} \in S, u \in R\left(v_{j}\right)} d\left(u, v_{j}\right) \leq \alpha$, we have:

(1) Every point $u$ is assigned to a cluster $C\left(s_{i}\right)$ such that $d\left(u, s_{i}\right) \leq \min _{s_{j} \in S} d\left(u, s_{j}\right)+2$ OPT using $O(n k \log (n / \delta))$ oracle queries with probability $1-O(\delta)$.

(2) APPROX-FARTHEST identifies a point $w$ in cluster $C\left(s_{i}\right)$ such that $\min _{v_{j} \in S} d\left(w, v_{j}\right) \geq \max _{v_{j} \in V} \min _{s_{j} \in S} d\left(v_{j}, s_{j}\right) /(6 \alpha / \mathrm{OPT}+3)$ with probability $1-O(\delta / k)$ using $O\left(|\widetilde{V}| \log ^{3}(n / \delta)\right)$ oracle queries.

Proof. (1) First, we argue that cores are calculated correctly. From Lemma 11.3, we have that a point $u \in C^{*}\left(s_{i}\right)$ is assigned to the center correctly $s_{i}$. Therefore, all the points from $\widetilde{V} \cap C^{*}\left(S_{i}\right)$ move to $C\left(S_{i}\right)$. As the size of $\left|C\left(S_{i}\right)\right| \geq\left|\widetilde{V} \cap C^{*}\left(S_{i}\right)\right| \geq 400 \log (n / \delta)$, we have $\left|R\left(s_{i}\right)\right| \geq 200 \log (n / \delta)$ with a probability $1-\left|C\left(s_{i}\right)\right|^{2} \delta / n^{2}$ (From Lemma 11.6). Using union bound, we have that all the cores are calculated 
correctly with a failure probability of $\sum_{i}\left|C\left(s_{i}\right)\right|^{2} / n^{2}=\delta$.

For every point, we compare the distance with every cluster center by maintaining a center that is the current closest. From Lemma 11.2 , we have that the query will fail with a probability of $\delta / n^{2}$. Using union bound, we have that the failure probability is $O\left(k n \delta / n^{2}\right)=\delta$. From Lemma 11.2, we have the approximation guarantee.

(2) From Lemma 11.10, we have our claim regarding the approximation guarantees. For ApPRox-FARTHEST, we use the parameters $t=2 \log (2 k / \delta), l=\sqrt{|\widetilde{V}|}$. As we make $O\left(|\widetilde{V}| \log ^{2}(k / \delta)\right)$ cluster comparisons using Algorithm CLUSTERCOMP (for Approx-FARTHEST), we have that the total number of oracle queries is $O\left(|\widetilde{V}| \log (n / \delta) \log ^{2}(k / \delta)\right)=O\left(|\widetilde{V}| \log ^{3}(n / \delta)\right)$. Using union bound, we have that the failure probability is $O\left(\delta / k+|\widetilde{V}| \log ^{2}(k / \delta) / n^{2}\right)=O(\delta / k)$.

THEOREM 11.12. [Theorem 4.4 restated] Given $p \leq 0.4$, a failure probability $\delta$, and $m=\Omega\left(\frac{\log ^{3}(n / \delta)}{\delta}\right)$. Then, Algorithm 7 achieves a $O(1)$-approximation for the $k$-center objective using $O\left(n k \log (n / \delta)+\frac{n^{2}}{m^{2}} k \log ^{2}(n / \delta)\right)$ oracle queries with probability $1-O(\delta)$.

Proof. Using similar proof as Lemma 10.1, we have that the approximation ratio of Algorithm 7 is $4(6 \alpha / \mathrm{OPT}+3)+2$. Using $\alpha=8 \mathrm{OPT}$ from Lemma 11.8, we have that the approximation factor is 206. For the first stage, from Lemma 11.11, we have that for all the $k$ iterations, the number of oracle queries is $O\left(|\widetilde{V}| k \log ^{3}(n / \delta)\right)$. Using union bound over $k$ iterations, success probability is $1-O(\delta)$. For the calculation of core, the query complexity is $O\left(|\widetilde{V}|^{2} k\right)$. For assignment, the query complexity is $O(n k \log (n / \delta))$. Therefore, total query complexity is $O\left(n k \log (n / \delta)+\frac{n}{m} k \log ^{4}(n / \delta)+\frac{n^{2}}{m^{2}} k \log ^{2}(n / \delta)\right)=O\left(n k \log (n / \delta)+\frac{n^{2}}{m^{2}} k \log ^{2}(n / \delta)\right)$.

\section{HIERARCHICAL CLUSTERING}

Lemma 12.1 (Lemma 5.1 Restated). Given a collection of clusters $C=\left\{C_{1}, \ldots, C_{r}\right\}$, our algorithm to calculate the closest pair (using Algorithm 4) identifies $C_{1}$ and $C_{2}$ to merge according to single linkage objective if $d_{S L}\left(C_{2}, C_{2}\right) \leq(1+\mu)^{3} \min _{C_{i}, C_{j} \in C} d\left(C_{i}, C_{j}\right)$ with $1-\delta$ probability and requires $O\left(r^{2} \log ^{2}(n / \delta)\right)$ queries.

Proof. In each iteration, our algorithm considers a list of $\left(\begin{array}{c}r \\ 2\end{array}\right)$ distance values and calculates the closest using Algorithm 4 . The claim follows from the proof of Theorem 3.6

Using the same analysis, we get the following result for complete linkage.

Lemma 12.2. Given a collection of clusters $C=\left\{C_{1}, \ldots, C_{r}\right\}$, our algorithm to calculate the closest pair (using Algorithm 4) identifies $C_{1}$ and $C_{2}$ to merge according to complete linkage objective if $d_{S L}\left(C_{2}, C_{2}\right) \leq(1+\mu)^{3} \min _{C_{i}, C_{j} \in C} d\left(C_{i}, C_{j}\right)$ with $1-\delta$ probability and requires $O\left(r^{2} \log ^{2}(n / \delta)\right)$ queries.

THEOREM 12.3 (THEOREM 5.2 RESTATED). In any iteration, suppose the distance between a cluster $C_{j} \in C$ and its identified nearest neighbor $\widetilde{C_{j}}$ is $\alpha$-approximation of its distance from the optimal nearest neighbor, then the distance between pair of clusters merged by Algorithm 11 is $\alpha(1+\mu)^{3}$ approximation of the optimal distance between the closest pair of clusters in $C$ with a probability of $1-\delta$ using $O\left(n \log ^{2}(n / \delta)\right)$ oracle queries.

Proof. Algorithm 11 iterates over the list of pairs $\left(C_{i}, \widetilde{C}_{i}\right), \forall C_{i} \in C$ and identifies the closest pair using Algorithm 4 . The claim follows from the proof of Theorem 3.6 